\title{
Efficacy and safety of plant-derived products for the treatment of osteoarthritis
}

\author{
This article was published in the following Dove Press journal: \\ Botanics:Targets and Therapy \\ 16 December 2014 \\ Number of times this article has been viewed
}

\section{Laura L Laslett \\ Xingzhong Jin \\ Graeme Jones}

Menzies Institute for Medical Research, University of Tasmania, Hobart, Tasmania, Australia
Correspondence: Laura L Laslett Menzies Institute for Medical Research, University of Tasmania, Private Bag 23, Hobart, Tasmania 7000, Australia

$\mathrm{Tel}+61362267736$

Fax +6I 362267704

Email laura.laslett@utas.edu.au
Background: Plant-derived therapies are traditionally used as medicines, but they have generally not been studied with the same rigor as pharmaceutical agents. This review summarizes the use of plant-derived products for osteoarthritis.

Methods: Sixty-three identified trials were summarized for pain, function, and safety outcomes using standardized mean differences (SMDs) and relative risks.

Results: Plant-derived therapies are effective for treating pain compared to placebo, as assessed using visual analog scores and numerical rating scales (SMD, 1.08; 95\% confidence interval [CI]: 0.72-1.44), or Western Ontario and McMaster University Osteoarthritis Index (WOMAC)/Knee injury and Osteoarthritis Outcome Score (KOOS) pain scales (SMD, 0.98; 95\% CI: 0.62-1.35). Classes demonstrating overall efficacy in more than one trial for either visual analog scores or WOMAC pain included Boswellia serrata, capsaicin, and ginger; there was single-trial evidence of the efficacy of another nine agents. Plant-derived therapies have similar efficacy to an active comparator (SMD, $0.32 ; P=0.08 ;-0.08 ; P=0.14$ ). Therapies are also effective for functional outcomes compared to placebo (SMD, 0.92; $P<0.001)$. However, significant heterogeneity remains for all pain and function outcomes, indicating that the results need to be interpreted with caution. Risk of adverse events was similar to placebo (relative risk $=1.13 ; P=0.1$ ), but reduced compared to an active comparator (relative risk, $0.75 ; P<0.001$ ).

Conclusion: Plant-derived therapies may be efficacious in treating osteoarthritic pain and functional limitations, and they appear to be safer than other active therapies. However, quality trials and long-term data are lacking, and the number of trials for each therapy is limited. Comparisons would be assisted by trial standardization.

Keywords: phytotherapy, plant extract, herbal, review, meta-analysis, osteoarthritis

\section{Introduction}

Osteoarthritis (OA) is the most common joint disorder and it predominantly affects the knees, hips, and hands of older adults. It is a leading cause of pain, functional limitations and disability worldwide, ${ }^{1}$ with levels of disability among people with OA having increased globally by over 25\% from 1990-2010. ${ }^{2}$ Despite the large disease burden, OA etiology is poorly understood, and treatment remains palliative. Commonly involved joint structures include subchondral bone, ligaments, menisci, periarticular muscles, peripheral nerves, and synovium. ${ }^{3,4}$

$\mathrm{OA}$ is no longer considered to be a single disease entity, but a collection of heterogeneous pathologies that result in a common outcome. ${ }^{5,6}$ The lack of a common causal pathway has hampered the development of effective treatments for modifying the natural history of the disease. Most existing treatments focus on relieving pain and 
improving function, and there are few examples of therapies that modify disease. The pathogenesis of pain in OA is complex and multifactorial, involving local nociception, inflammatory mediators, and central sensitization..$^{5-8}$

Treatment of osteoarthritic pain includes a wide range of therapies, from: nonpharmacological treatments (eg, education, weight reduction, physiotherapy); pain medications (eg, paracetamol, nonsteroidal anti-inflammatory drugs, opioids); nutraceuticals (eg, glucosamine, chondroitin sulfate); and surgical therapies (eg, joint replacement). Additionally, the effect sizes (ES) of existing treatments vary, but they are typically small to moderate ${ }^{9}$ and fall short of the levels of pain relief desired by patients. ${ }^{10}$ Medicinal plants form the basis of traditional medicinal systems around the world, and the number and type of botanically-based therapies and their mechanisms of action are similarly diverse. Given the limited efficacy of many existing treatments, there is considerable scope for alternative therapies, and plant-based therapies are well-placed to supplement this gap.

Additionally, controversy surrounding use of cyclooxygenase-2 inhibitors and heightened cardiovascular risk, ${ }^{11-14}$ highlights the importance of finding safer treatment options to minimize adverse side effects. ${ }^{15}$ Botanical treatments may play a role in treatment of OA even if they are only moderately effective if they also have favorable safety profiles compared to alternatives (eg, nonsteroidal anti-inflammatory drugs). Additionally, given the high proportion of persons with OA using complementary and alternative medicines of various types, ${ }^{16,17}$ assessment of treatment efficacy and the relative risk (RR) of side effects is warranted.

The efficacy and safety of plant-based therapies for OA have been the subject of several previous reviews. ${ }^{18-21}$ However, the number of studies trialing therapies is steadily increasing, necessitating more recent reviews; no previous reviews have summarized trials in such a way that efficacy and safety are directly comparable, either to placebo or to an active comparator.

Therefore, this review investigates the efficacy and safety of plant-derived products for the treatment of OA, as compared to placebo and active comparators, on OA pain and function.

\section{Methods}

\section{Identification of clinical trials}

Literature databases (PubMed and Embase) were searched for randomized controlled trials of botanical therapies as an intervention for pain or functional outcomes in OA, where the comparator was a placebo or an active comparator. The following keywords were used: "phytotherapy OR medicinal plants OR plant extract OR herbal"; "osteoarthritis" (both as a single phrase and as a topic) and "hip" or "knee" or "hand"; "randomized controlled trial [publication type]" or "controlled clinical trial [publication type]"; and "humans" that were published up to June 2013. This was supplemented by manually searching the bibliographies of relevant published reviews and papers.

Database searches identified a total of 144 studies: 92 in PubMed and 104 in Embase, and 52 in both. This yielded 58 studies after unsuitable trials were excluded. Supplemented papers included one notable plant-based treatment class, which did not appear in the original search (capsaicin) and an article using pine bark, which was not indexed under plant-based therapies. ${ }^{22}$

\section{Inclusion/exclusion criteria}

The included studies were randomized controlled trials of at least one plant-based therapy conducted with humans, where at least a subpopulation of adult patients had OA, as long as this subpopulation was presented separately. Studies were excluded if they were observational studies, not in English, where the botanical therapy was not the subject of the trial, where the botanical therapy was in both active and control medications (but no additional botanical therapy was used as an intervention), and when insufficient data were reported to extract ES (eg, where medians rather than means were reported). Topical therapies were included. Studies on animal populations and in participants with back pain or spinal OA were excluded. Studies were read by one reviewer (LLL).

\section{Definition of plant-derived products}

Treatments were included if they were any type of plantderived intervention (defined as any plant preparation, including whole, powder, extract, or standardized mixture), and they were excluded if there was any preparation of synthetic origin. These treatments could be used in any way, but they are typically ingested orally or applied topically on the skin (Table 1).

Treatments could be compared to an inert substance (placebo) or an active comparator. Botanical therapies used in conjunction with other treatments or combined with a nonbotanical substance were also included if the effect of the nonherbal intervention was consistent among all groups and was quantifiable. Treatment arms were omitted if they were additional to active versus placebo or active versus active comparator comparisons. 


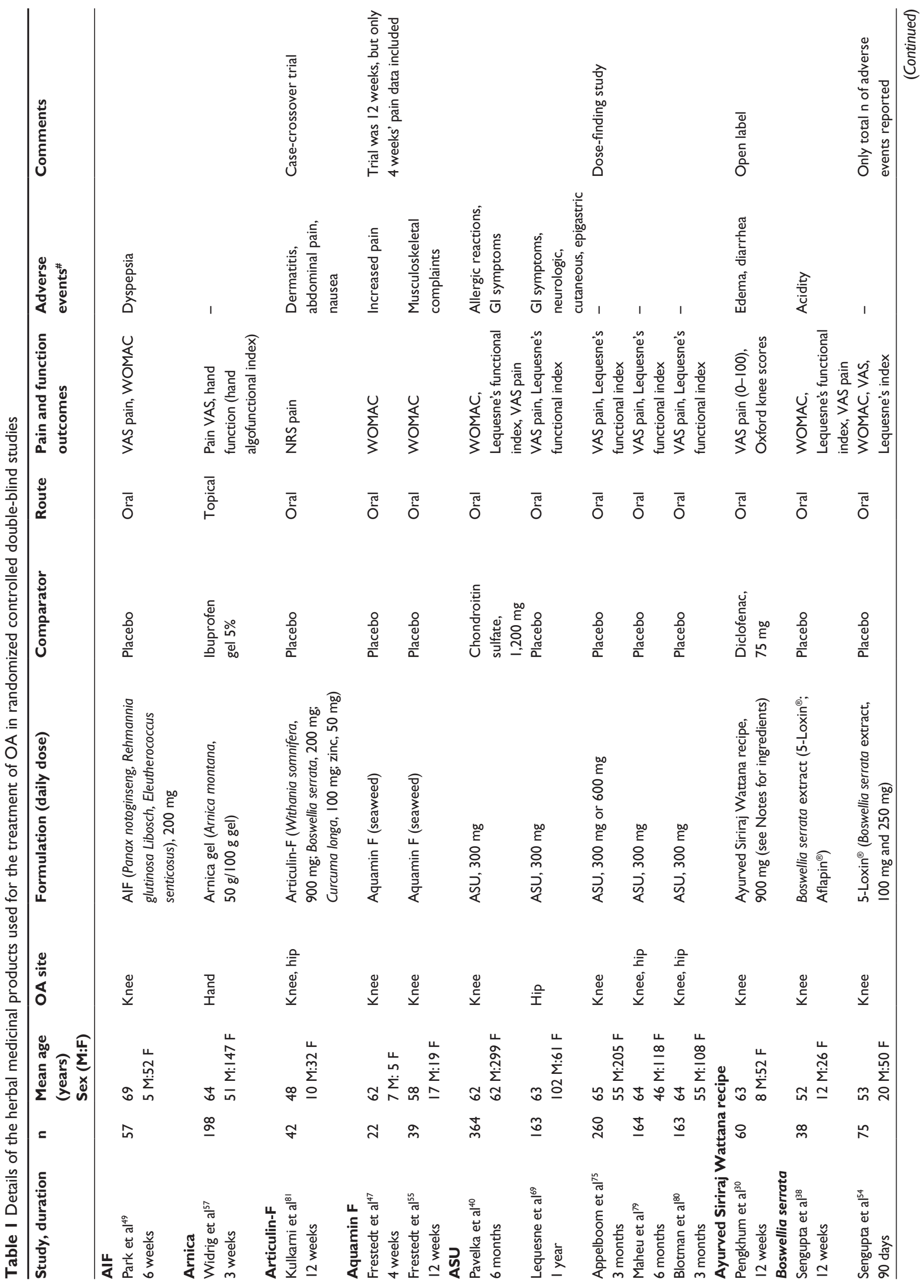




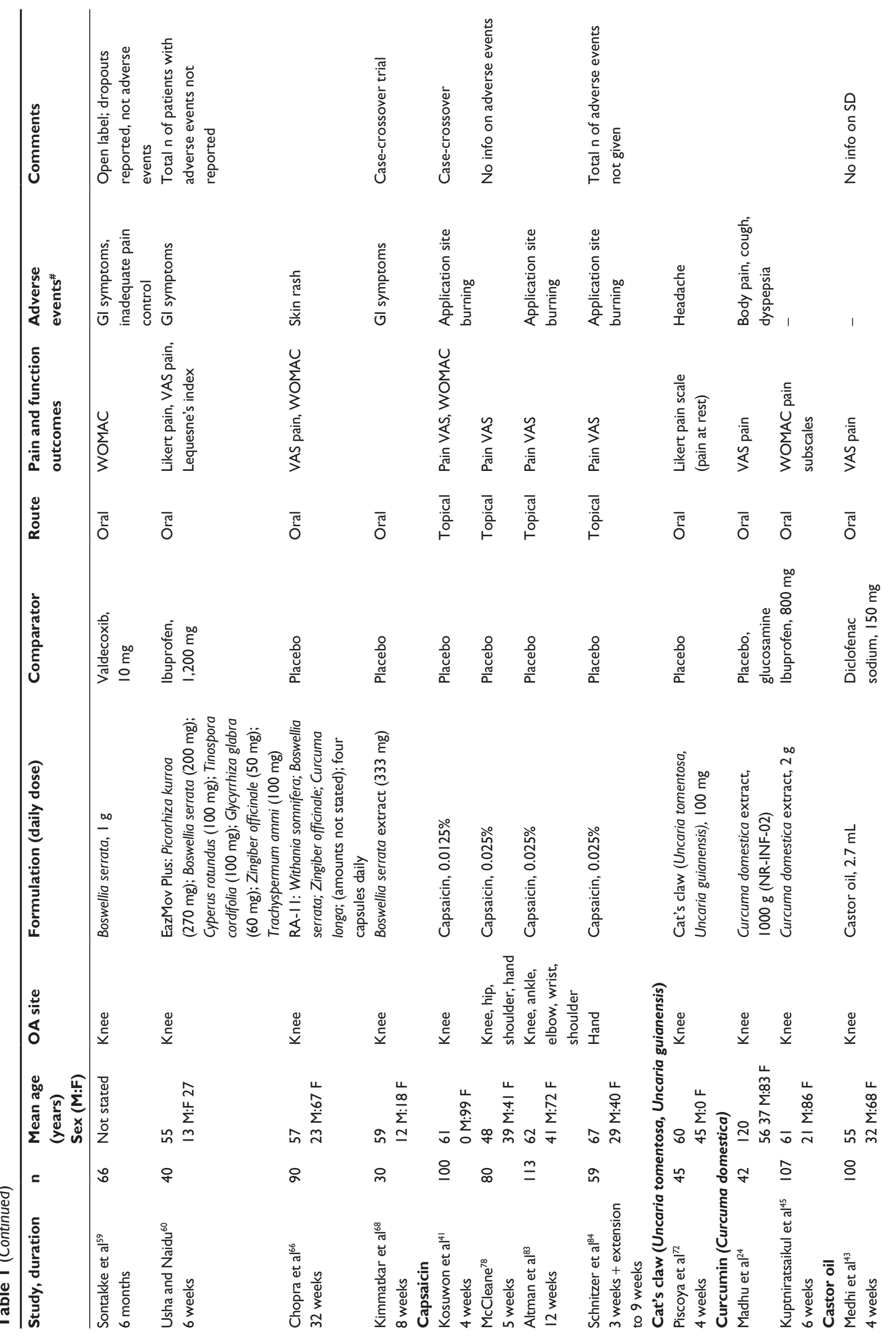



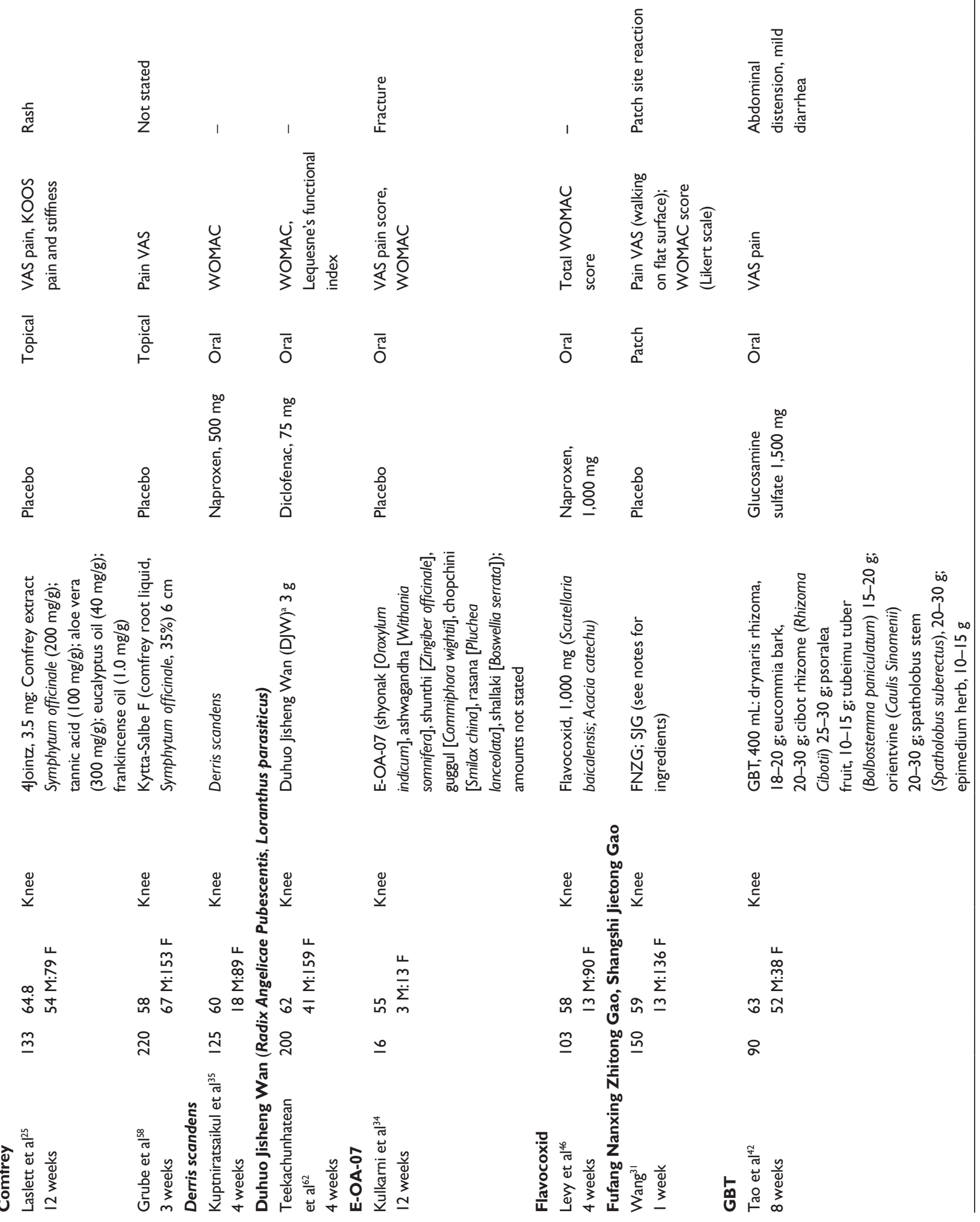


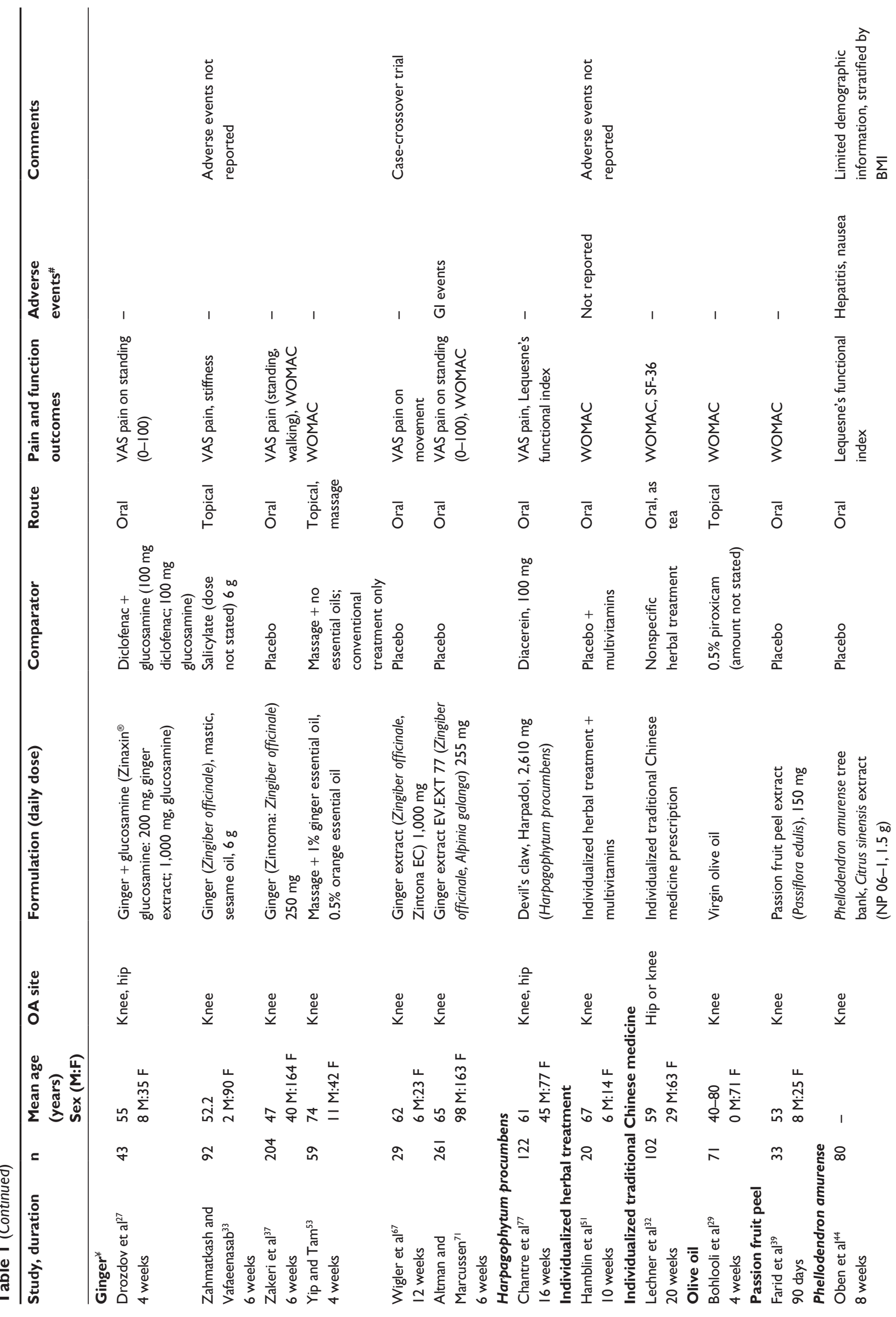



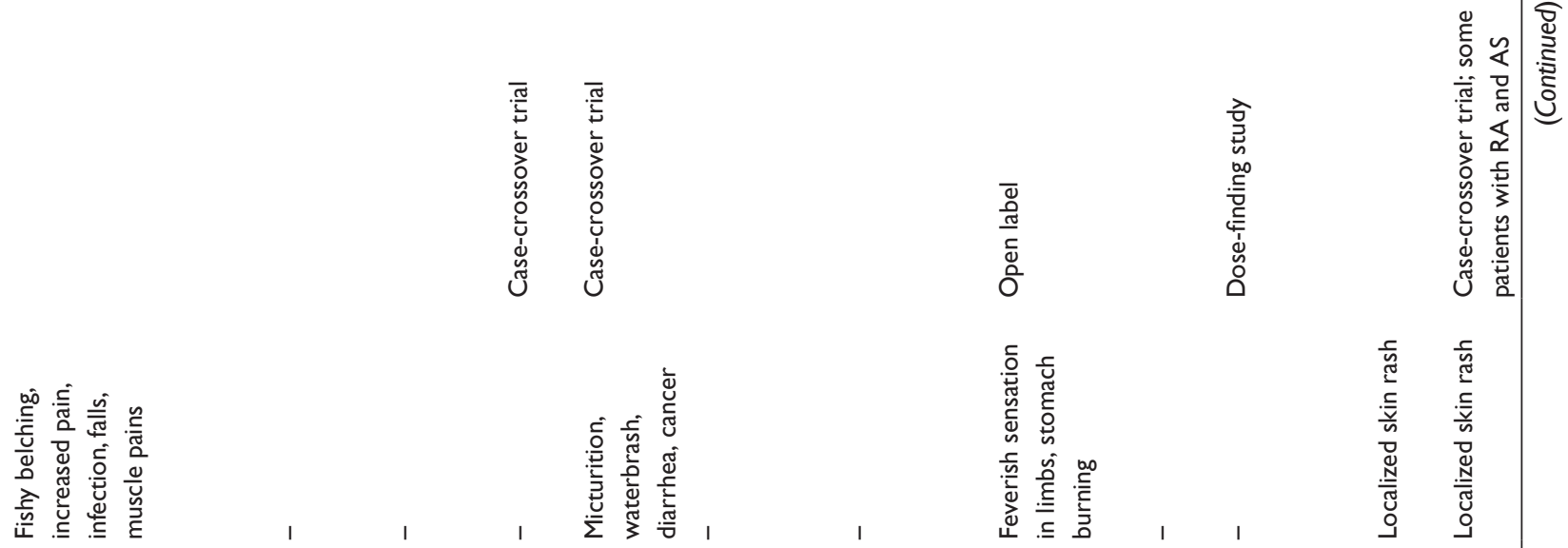

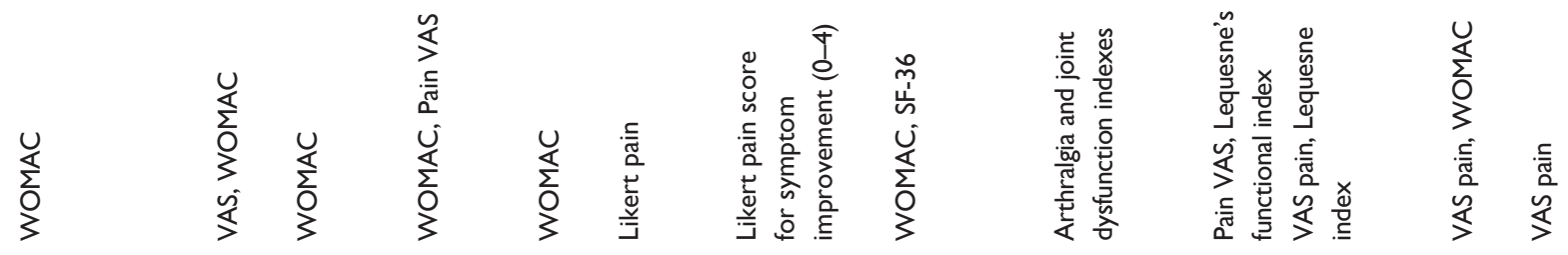

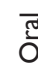

ठ

व

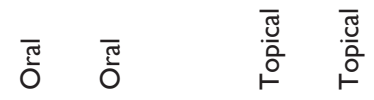

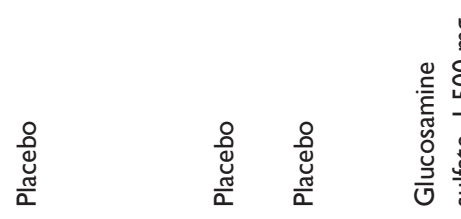

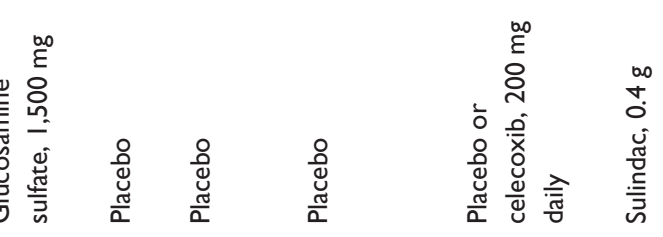

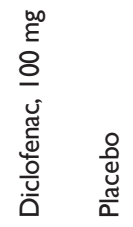

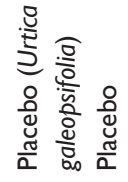

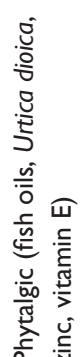

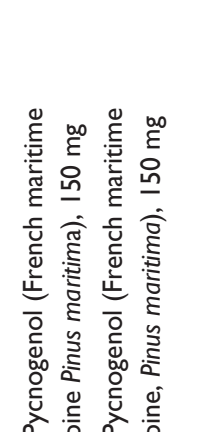

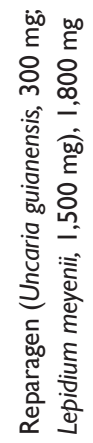
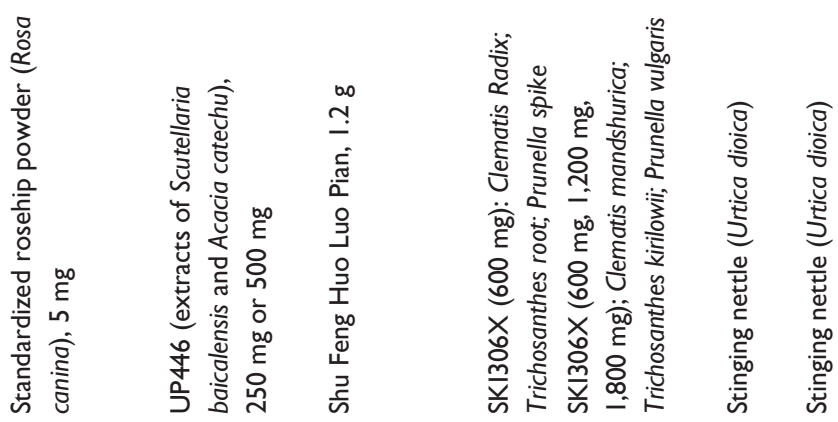

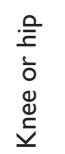
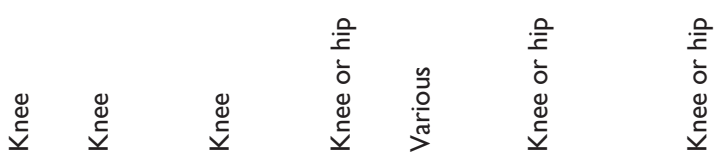

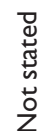

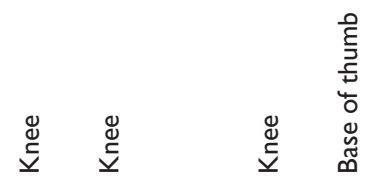

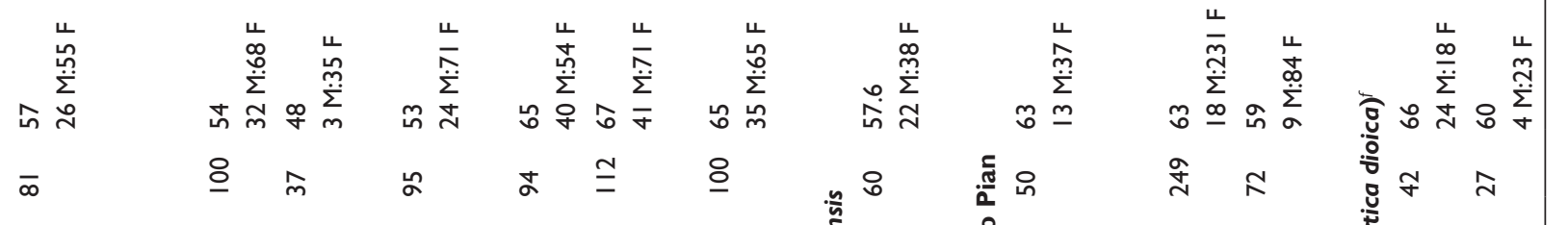

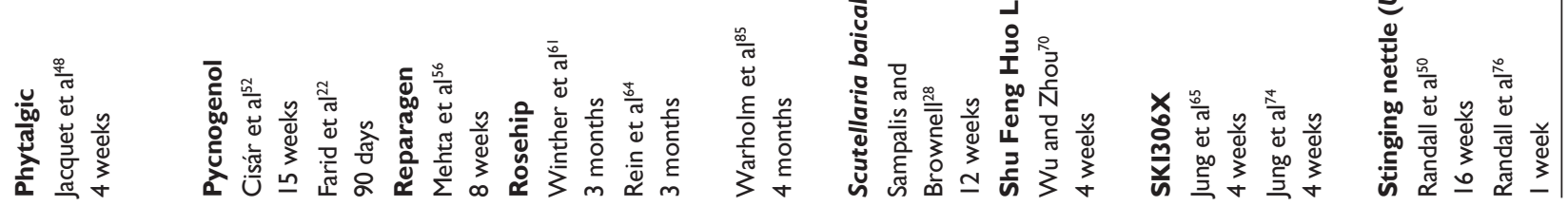




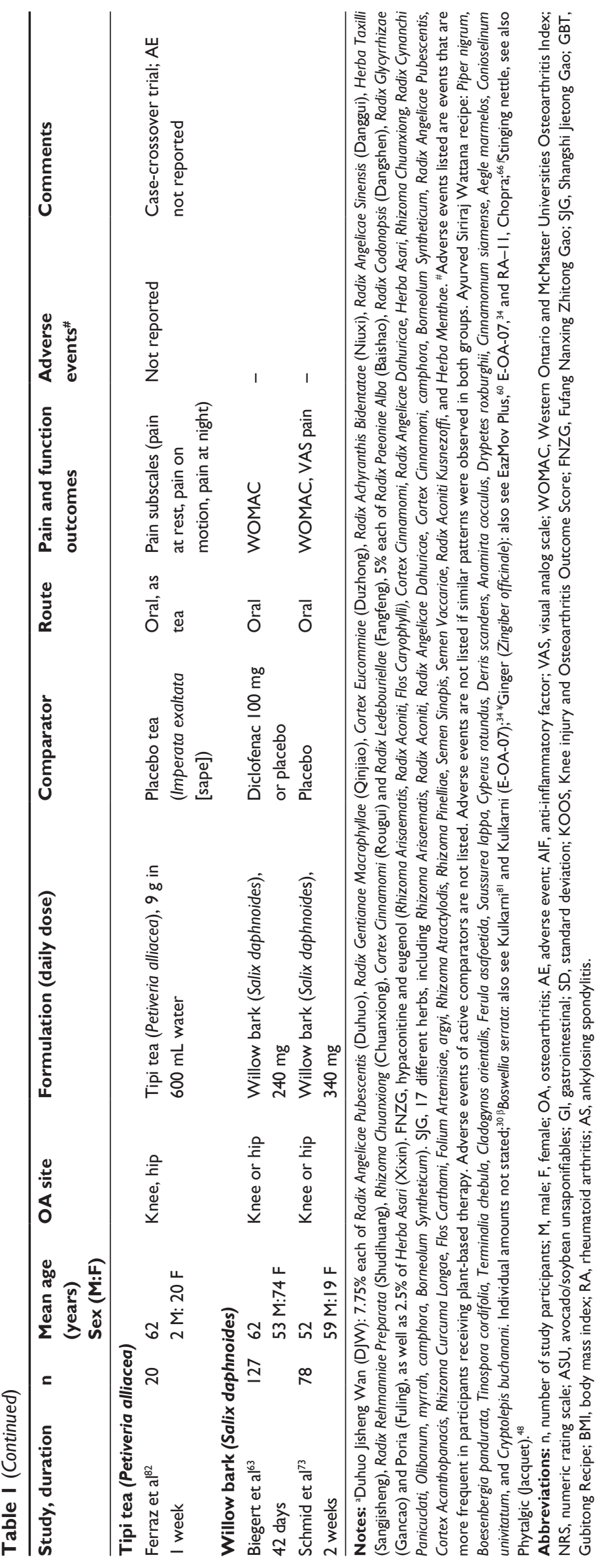




\section{Data extraction and quality assessment}

Data relating to treatment duration, demographic information, OA site, route, intervention(s), the patient-rated outcomes of pain and function, ES, and adverse events were extracted into predefined tables by one author (LLL).

Methodological quality was assessed using The Cochrane Collaboration's tool for assessing risk of bias by one author (XJ). ${ }^{23}$ Studies were assessed as having a low risk of bias, unclear risk of bias, or a high risk of bias. Included domains were: random sequence generation; allocation concealment; blinding of participants and personnel; blinding of outcome assessment; incomplete outcome data; selective reporting; and other sources of bias (scoring for individual items is shown in Table 2). Scores were summed to create a risk of bias score (possible range: 0-14), with higher scores indicating a greater risk of bias. Studies were also scored as to whether or not they required participants to cease pain medications prior to trial entry (yes/no).

\section{Identified trials}

Table 1 shows the 63 double-blind randomized controlled trials of therapies of botanical origin to treatment of pain in OA..$^{22,24-85}$ The 63 studies include eight case-crossover clinical trials. ${ }^{41,61,64,67,68,76,81,82}$ Treatment duration ranged from 1 week-1 year. Inclusion and exclusion criteria varied between trials, but patients were typically required to have at least moderate pain and either radiological evidence of OA or to be clinically diagnosed as having OA, or both. Where a study was defined as a case-crossover trial, data were extracted only up to the point of crossover, so that the data could be compared with those derived from parallel trials.

\section{Outcome assessment}

Pain outcomes included individual pain intensity scores assessed using a visual analog scale ([VAS], continuous data ranging from $0-100$ ) or a numeric rating scale ([NRS]; integers), or a Likert scale (numbers representing descriptions, eg, "never", "sometimes", "often"), and data from the pain scales of pain and function questionnaires (Western Ontario and McMaster Universities Osteoarthritis Index [WOMAC] ${ }^{86}$ and the Knee injury and Osteoarthritis Outcome Score $[\mathrm{KOOS}]^{87}$ ). Data were used from total pain scores where possible.

Rasch analyses of the WOMAC pain subscale have previously suggested that it measures a combined function-pain construct. ${ }^{88,89}$ Function outcomes included the KOOS symptom score,${ }^{87}$ the WOMAC function score ${ }^{86}$ and the Lequesne's functional index. ${ }^{90}$ Adverse events included the total number of patients with one or more adverse events.
Trials that did not have data for any of the above categories did not contribute data to this review.

\section{Statistics}

Data were analyzed using the "metan" command in Stata 12.1 (StataCorp LP, College Station, TX, USA). Statistical significance was set as a $P$-value $\leq 0.05$ (two-tailed).

The main analyses were performed using a random effects model that generated an estimate of ES (standardized mean difference [SMD]). This is calculated by dividing the mean difference between treatments by the standard deviation (SD) of the difference. It is, therefore, a number without units that can be used for cross-study comparisons. Clinically, ES $=0.2$ is considered small, $\mathrm{ES}=0.5$ is moderate, and $\mathrm{ES}>0.8$ is a large effect. ${ }^{9}$ These were pooled using the method of DerSimonian and Laird. ${ }^{91}$

Subgroup analyses were analyzed using a random effects model if there was significant heterogeneity and fixed effects model if there was not. Fixed effects models were weighted using the inverse of the variance of the difference in means. All estimates of heterogeneity were taken from the MantelHaenszel model. Associations between the risk of bias score and ES were assessed using the Spearman's rank correlation coefficients. Adverse event data were summarized using both fixed and random effects. The direction of the effect of KOOS outcome data was reversed to meaningfully pool it with WOMAC data.

A change in means was calculated using the final result minus the baseline result. Standard error or SD for the change in means was obtained from original papers where available. Where unavailable, the standard error of the difference was calculated using the following formula,

$\sqrt{ }\left(\mathrm{SE}_{\text {Baseline }}{ }^{2}+\mathrm{SE}_{\text {Follow-up }}{ }^{2}-2 \times r \times \mathrm{SE}_{\text {Baseline }} \times \mathrm{SE}_{\text {Follow-up }}\right)$

where baseline and follow-up are the first and last time points, and $r$ is the correlation between standard errors, conservatively assumed to be 0.7 . Adverse event data were assessed using RR.

\section{Results}

Summaries of the 63 included trials are presented in Table 1.24-84 These studies encompass a wide range of botanical therapies administered orally, topically, and by other methods. Treatments were predominantly conducted on OA of the knee, but they also included OA of the hand and hip. Results are presented for pain outcomes (VAS, NRS pain scores, and WOMAC and KOOS pain scales), function, and adverse events data, and are presented separately by comparator (placebo or active control). Data were summarized by 
Table 2 Risk of bias assessment for individual randomized controlled trials of botanical therapy versus placebo or active comparator

\begin{tabular}{|c|c|c|c|c|c|c|c|c|}
\hline Study & Treatment & $\begin{array}{l}\text { Random } \\
\text { sequence } \\
\text { generation }\end{array}$ & $\begin{array}{l}\text { Allocation } \\
\text { concealment }\end{array}$ & $\begin{array}{l}\text { Blinding: } \\
\text { participants }\end{array}$ & $\begin{array}{l}\text { Blinding: } \\
\text { outcome } \\
\text { assessment }\end{array}$ & $\begin{array}{l}\text { Incomplete } \\
\text { outcome } \\
\text { data }\end{array}$ & $\begin{array}{l}\text { Selective } \\
\text { reporting }\end{array}$ & Other \\
\hline Madhu et $\mathrm{al}^{24}$ & Curcumin & Low risk & Low risk & Low risk & Low risk & Low risk & Low risk & Low risk \\
\hline Bohlooli et $\mathrm{al}^{29}$ & Olive oil & Low risk & Low risk & Low risk & Low risk & Risk unclear & Low risk & Risk unclear \\
\hline Drozdov et $\mathrm{al}^{27}$ & Ginger & High risk & Risk unclear & Risk unclear & Risk unclear & Low risk & Low risk & Low risk \\
\hline Laslett et $\mathrm{al}^{25}$ & 4Jointz & Low risk & Low risk & Low risk & Low risk & Low risk & Low risk & Low risk \\
\hline Niempoog et $\mathrm{al}^{26}$ & Ginger & Risk unclear & Risk unclear & Low risk & Low risk & Risk unclear & Low risk & Low risk \\
\hline Pengkhum et $\mathrm{al}^{30}$ & $\begin{array}{l}\text { Ayurved Siriraj } \\
\text { Wattana }\end{array}$ & Low risk & Risk unclear & High risk & High risk & Low risk & Low risk & Low risk \\
\hline Sampalis and Brownell ${ }^{28}$ & UP446 & Risk unclear & Risk unclear & Risk unclear & Risk unclear & Risk unclear & Low risk & Low risk \\
\hline Wang et $\mathrm{al}^{31}$ & FNZG, SJG & Low risk & Low risk & Low risk & Low risk & Low risk & Low risk & Low risk \\
\hline Kosuwon et $\mathrm{al}^{41}$ & Capsaicin & Low risk & High risk & Risk unclear & Risk unclear & Low risk & Low risk & Low risk \\
\hline Kulkarni, 20II I4 & E-OA-07 & Risk unclear & Low risk & Risk unclear & Risk unclear & High risk & Low risk & Low risk \\
\hline Kuptniratsaikul et $\mathrm{al}^{35}$ & Derris scandens & Low risk & Low risk & High risk & Risk unclear & Risk unclear & Low risk & Low risk \\
\hline Lechner et $\mathrm{al}^{32}$ & $\mathrm{TCM}$ & Low risk & Low risk & Low risk & Low risk & Low risk & Low risk & Low risk \\
\hline Vishal et $\mathrm{al}^{36}$ & Boswellia serrata & Low risk & Low risk & Low risk & Low risk & Risk unclear & Low risk & Low risk \\
\hline $\begin{array}{l}\text { Zahmatkash and } \\
\text { Vafaeenasab }^{33}\end{array}$ & Ginger & Low risk & Risk unclear & Risk unclear & Risk unclear & Risk unclear & Low risk & Low risk \\
\hline Zakeri et $\mathrm{al}^{37}$ & Ginger & Risk unclear & Risk unclear & Low risk & Low risk & Risk unclear & Low risk & Low risk \\
\hline Farid et $\mathrm{al}^{39}$ & $\begin{array}{l}\text { Passion fruit } \\
\text { peel }\end{array}$ & Risk unclear & Risk unclear & Low risk & Low risk & Risk unclear & Low risk & Low risk \\
\hline Pavelka et $\mathrm{al}^{40}$ & ASU & Low risk & Risk unclear & Low risk & Low risk & Low risk & Low risk & Low risk \\
\hline Sengupta et $\mathrm{al}^{38}$ & Boswellia serrata & Low risk & Low risk & Low risk & Low risk & Low risk & Low risk & Low risk \\
\hline Frestedt et $\mathrm{al}^{47}$ & Aquamin F & Low risk & Low risk & Low risk & Low risk & High risk & Low risk & Risk unclear \\
\hline Jacquet et $\mathrm{al}^{48}$ & Phytalgic & Low risk & Low risk & Low risk & Low risk & Low risk & Low risk & Low risk \\
\hline Kuptniratsaikul et al ${ }^{45}$ & Curcumin & Low risk & Low risk & High risk & High risk & Risk unclear & Low risk & Low risk \\
\hline Levy et $\mathrm{al}^{46}$ & Flavocoxid & Risk unclear & Risk unclear & Risk unclear & Risk unclear & Low risk & Low risk & Low risk \\
\hline Medhi et $\mathrm{al}^{43}$ & Castor oil & Risk unclear & Risk unclear & Risk unclear & Risk unclear & Risk unclear & Low risk & Low risk \\
\hline Oben et $\mathrm{al}^{44}$ & $\begin{array}{l}\text { Phellodendron } \\
\text { amurense }\end{array}$ & Low risk & Risk unclear & Low risk & Low risk & Risk unclear & Low risk & Low risk \\
\hline Park et al ${ }^{49}$ & AIF & Risk unclear & Low risk & Low risk & Low risk & High risk & Low risk & Low risk \\
\hline Tao et $\mathrm{al}^{42}$ & GBT & High risk & Risk unclear & High risk & High risk & Low risk & Low risk & Low risk \\
\hline Cisár et $\mathrm{al}^{52}$ & Pycnogenol & Risk unclear & Low risk & Low risk & Low risk & Low risk & Low risk & Low risk \\
\hline Frestedt et $\mathrm{a}^{55}$ & Aquamin $\mathrm{F}$ & Low risk & Low risk & Low risk & Low risk & High risk & Low risk & Low risk \\
\hline Hamblin et $\mathrm{al}^{51}$ & $\begin{array}{l}\text { Individualized } \\
\text { herbal } \\
\text { treatment }\end{array}$ & Risk unclear & Low risk & Risk unclear & Risk unclear & Low risk & Low risk & Low risk \\
\hline Randall et $\mathrm{al}^{50}$ & Stinging nettle & Low risk & Low risk & Low risk & Low risk & Risk unclear & Low risk & Low risk \\
\hline Sengupta et $\mathrm{al}^{54}$ & Boswellia serrata & Low risk & Low risk & Low risk & Low risk & Low risk & Low risk & Low risk \\
\hline Yip and $\mathrm{Tam}^{53}$ & $\begin{array}{l}\text { Massage }+ \\
\text { essential oils }\end{array}$ & High risk & High risk & High risk & High risk & Low risk & Low risk & Low risk \\
\hline Grube et $\mathrm{al}^{58}$ & Comfrey & Risk unclear & Risk unclear & Risk unclear & Risk unclear & Risk unclear & Low risk & Low risk \\
\hline Mehta et $\mathrm{al}^{56}$ & Reparagen & Low risk & Low risk & Low risk & Low risk & Risk unclear & Low risk & Low risk \\
\hline Sontakke et al ${ }^{59}$ & Boswellia serrata & Low risk & Risk unclear & High risk & High risk & Risk unclear & Risk unclear & Low risk \\
\hline Widrig et $\mathrm{al}^{57}$ & Arnica gel & Low risk & Low risk & Low risk & Low risk & Low risk & Low risk & Low risk \\
\hline Farid et $\mathrm{al}^{22}$ & Pine bark & Risk unclear & Risk unclear & Low risk & Low risk & Low risk & Low risk & Low risk \\
\hline Usha and Naidu ${ }^{60}$ & EazMov Plus & Low risk & Risk unclear & High risk & High risk & Low risk & Low risk & Low risk \\
\hline Winther et $\mathrm{al}^{61}$ & Rosehip & Low risk & Low risk & Low risk & Low risk & Risk unclear & Low risk & Low risk \\
\hline Biegert et $\mathrm{al}^{63}$ & Willow bark & Low risk & Low risk & Low risk & Low risk & Risk unclear & Low risk & Low risk \\
\hline Chopra et $\mathrm{al}^{66}$ & RA-II & Low risk & Low risk & Low risk & Low risk & Risk unclear & Low risk & Low risk \\
\hline Jung et $\mathrm{al}^{65}$ & SKI306X & Low risk & Low risk & High risk & High risk & Risk unclear & Low risk & Low risk \\
\hline Rein et $\mathrm{al}^{64}$ & Rosehip & Low risk & Low risk & Low risk & Low risk & Low risk & Low risk & Low risk \\
\hline Teekachunhatean et al ${ }^{62}$ & DJW & Risk unclear & Risk unclear & Low risk & Low risk & Risk unclear & Low risk & Low risk \\
\hline Kimmatkar et $\mathrm{al}^{68}$ & Boswellia serrata & Low risk & Risk unclear & Low risk & Low risk & Risk unclear & Low risk & Low risk \\
\hline Wigler et $\mathrm{al}^{67}$ & Ginger & Low risk & Low risk & Low risk & Low risk & Risk unclear & Low risk & Low risk \\
\hline Lequesne et $a^{69}$ & ASU & Low risk & Low risk & Low risk & Low risk & Risk unclear & Low risk & Low risk \\
\hline
\end{tabular}


Table 2 (Continued)

\begin{tabular}{|c|c|c|c|c|c|c|c|c|}
\hline Study & Treatment & $\begin{array}{l}\text { Random } \\
\text { sequence } \\
\text { generation }\end{array}$ & $\begin{array}{l}\text { Allocation } \\
\text { concealment }\end{array}$ & $\begin{array}{l}\text { Blinding: } \\
\text { participants }\end{array}$ & $\begin{array}{l}\text { Blinding: } \\
\text { outcome } \\
\text { assessment }\end{array}$ & $\begin{array}{l}\text { Incomplete } \\
\text { outcome } \\
\text { data }\end{array}$ & $\begin{array}{l}\text { Selective } \\
\text { reporting }\end{array}$ & Other \\
\hline Wu and $\mathrm{Zhou}^{70}$ & $\begin{array}{l}\text { Shu Feng Huo } \\
\text { Luo Pian }\end{array}$ & Risk unclear & Risk unclear & Risk unclear & Risk unclear & High risk & Risk unclear & Risk unclear \\
\hline Altman and Marcussen ${ }^{71}$ & Ginger & Low risk & Low risk & Low risk & Low risk & High risk & Low risk & Low risk \\
\hline Appelboom et $\mathrm{al}^{75}$ & ASU & Risk unclear & Risk unclear & Risk unclear & Risk unclear & Low risk & Low risk & Low risk \\
\hline Jung and Roh ${ }^{74}$ & SKI306X & Risk unclear & Risk unclear & Low risk & Low risk & Risk unclear & Low risk & Low risk \\
\hline Piscoya et al ${ }^{72}$ & Cat's claw & Risk unclear & Risk unclear & Low risk & Low risk & Low risk & Low risk & Low risk \\
\hline Schmid et $\mathrm{al}^{73}$ & Willow bark & Low risk & Low risk & Low risk & Low risk & Low risk & Low risk & Low risk \\
\hline Chantre et $\mathrm{al}^{77}$ & Devil's claw & Low risk & Low risk & Low risk & Low risk & Low risk & Low risk & Low risk \\
\hline Randall et $\mathrm{al}^{76}$ & Stinging nettle & Risk unclear & Risk unclear & Low risk & Low risk & Low risk & Low risk & Low risk \\
\hline McCleane ${ }^{78}$ & Capsaicin & Low risk & Low risk & Low risk & Low risk & High risk & Low risk & Low risk \\
\hline Maheu et $\mathrm{al}^{79}$ & ASU & Low risk & Low risk & Low risk & Low risk & Risk unclear & Low risk & Low risk \\
\hline Blotman et $\mathrm{al}^{80}$ & ASU & Risk unclear & Risk unclear & Low risk & Low risk & Risk unclear & Low risk & Low risk \\
\hline Altman et $\mathrm{al}^{83}$ & Capsaicin & Risk unclear & Risk unclear & Risk unclear & Risk unclear & High risk & Low risk & Low risk \\
\hline Schnitzer et $\mathrm{al}^{84}$ & Capsaicin & Risk unclear & Risk unclear & Risk unclear & Low risk & High risk & Low risk & Low risk \\
\hline Ferraz et $\mathrm{al}^{82}$ & Tipi tea & Risk unclear & High risk & Risk unclear & Risk unclear & Risk unclear & Risk unclear & Risk unclear \\
\hline Kulkarni et $\mathrm{al}^{81}$ & Articulin-F & Risk unclear & Risk unclear & Low risk & Low risk & Risk unclear & Low risk & Low risk \\
\hline
\end{tabular}

Abbreviations: FNZG, Fufang Nanxing Zhitong Gao; SJG, Shangshi Jietong Gao; TCM, traditional Chinese medicine; ASU, avocado/soybean unsaponifiables; AIF, antiinflammatory factor; GBT, Gubitong Recipe; DJW, Duhuo Jisheng Wan.

year of publication and compared using SMDs as compared to placebo or an active comparator. An active comparator is typically a nonsteroidal anti-inflammatory agent, but it also includes any other osteoarthritic therapy.

\section{Efficacy}

\section{Pain efficacy compared to placebo}

There were sufficient numbers of studies to present data on five subgroups of plant-based therapies: comfrey and comfrey blends; $;{ }^{25,58}$ Boswellia serrata extracts and blends; ${ }^{36,38,54,66,68,81}$ capsaicin; ${ }^{41,78,83,84}$ avocado/soybean unsaponifiables (ASUs), ${ }^{69,75,79,80}$ ginger; ${ }^{37,53,71}$ and pine bark ${ }^{22,52}$ (Figures 1 and 2).

For pain, as assessed by the VAS, NRS, and Likert pain scores (Figure 1), the SMDs (given using random effects) for subgroups are: comfrey, 1.70 (95\% CI: -0.82 to 4.22 ; $P=0.2$ ); Boswellia serrata, 1.33 (95\% CI: 0.74-1.92; $P<0.001$ ); capsaicin 0.48 (95\% CI: $0.27-0.70 ; P \leq 0.001$ ); and ASUs, 1.09 ( $95 \% \mathrm{CI}:-0.08$ to $2.25 ; P=0.068$ ). The SMD for the remaining botanical therapies is 0.94 (95\% CI: 0.48 $1.40 ; P<0.001)$. This would imply a large benefit from all classes of plant-based therapies (apart from capsaicin, which was moderate) on pain scores (as assessed by VAS and NRS) compared to placebo. Therefore, Boswellia serrata, capsaicin, and the ungrouped treatments as a whole are efficacious, but SMDs for comfrey and ASUs are not (SMD, 1.70; $P=0.18 ; \mathrm{SMD}, 1.09 ; P=0.068$ ). There was no association between ES and risk of bias $(\rho=-0.01 ; P=0.94)$.
Heterogeneity existed for plant-based therapies as a whole $\left(I^{2}=93.5 \%\right)$ and for all subgroups except capsaicin, with $I^{2}$ values of $98.9 \%$ for comfrey, $85.2 \%$ for Boswellia serrata, and $97.9 \%$ for ASUs. Individual trials of plant-based therapies demonstrating significant benefit over placebo include: NR-INF-02 (Turmacin ${ }^{\mathrm{TM}}$, Curcuma longa); ${ }^{24}$ pine bark extract (Pycnogenol ${ }^{\circledR}$ ); 52 SKI306X (extract of Clematis mandshurica, Trichosanthes kirilowii, and Prunella vulgaris) $;{ }^{74} \mathrm{E}-\mathrm{OA}-\mathrm{O} 7$ (Lanconone $^{\mathrm{TM}}$, extract of shyonaka [Oroxylum indicum]; ashwagandha [Withania somnifera]; shunthi [Zingiber officinale]; guggul [Commiphora wightii]; chopchini [Smilax china]; rasana [Pluchea lanceolata]; shallaki [Boswellia serrata]); ${ }^{34}$ and willow bark (Salix purpurea $x$ daphnoides). ${ }^{73}$

Studies using anti-inflammatory factor ([AIF]; extract of Panax notoginseng [Burk] F H Chen, Rehmannia glutinosa Libosch, and Eleutherococcus senticosus), ${ }^{49}$ rosehip (Rosa canbina $)^{64}$ and stinging nettle (Urtica dioica) ${ }^{76} \mathrm{did}$ not reach statistical significance. These results are also summarized in Table 3.

For WOMAC and KOOS pain scores (Figure 2), the SMD for Boswellia serrata extracts and blends is 4.21 (95\% CI: $1.85-6.57 ; P<0.001$ ), with considerable heterogeneity. All trials required patients to cease pain medications. Later trials had substantially smaller ES, though all are large. The SMD for ginger-based therapies is 0.28 (95\% CI: $0.10-0.46 ; P=0.002)$, without significant heterogeneity. However, the two trials, not including massage, had much 
higher SMDs, both of which required participants to cease pain medications prior to study commencement ${ }^{37,71}$ and, therefore, might inflate the ES - one of which ${ }^{71}$ had a high risk of bias due to the incomplete assessment of outcomes. The SMD for pine bark was 0.74 (95\% CI: -1.03 to 2.50 ; $P=0.41)$, with significant heterogeneity $\left(I^{2}=94.2 \%\right)$. There was no association between risk of bias and ES $(\rho=-0.01$; $P=0.95)$. The overall SMD for the unclassified therapies was 0.40 (95\% CI: $0.11-0.70 ; P=0.007)$, but with significant heterogeneity between studies $\left(I^{2}=79.6 \%\right)$. Individual agents that demonstrated significant benefit over placebo included the following: capsaicin; ${ }^{41}$ UP446 (a blend of extracts of Scutellaria baicalensis and Acacia catechu); ${ }^{28}$ E-OA-O7 (extract of shyonak (Oroxylum indicum); ashwagandha (Withania somnifera); shunthi (Zingiber officinale); guggul (Commiphora wightii); chopchini (Smilax china); rasana (Pluchea lanceolata); shallaki (Boswellia serrata); ${ }^{34}$ passion fruit peel (Passiflora edulis); ${ }^{39}$ and Phytalgic ${ }^{\circledR}$ (a combination of stinging nettle Urtica dioica and fish oil). ${ }^{48}$

Randomized controlled trials of compounds containing traditional Chinese ingredients, ${ }^{31,32}$ individualized herbal treatment ${ }^{51}$ or mineral supplements, ${ }^{47,55}$ comfrey (Symphytum officinale), ${ }^{25}$ AIF (Panax notoginseng, Rehmannia glutinosa Libosch, Eleutherococcus senticosus), ${ }^{49}$ a trial of stinging nettle (Urtica dioica) alone, ${ }^{50}$ and rosehip (Rosa canina $)^{61}$ did not demonstrate efficacy on WOMAC or KOOS pain scales when compared to placebo.

Overall, plant-based therapies including Boswellia serrata, capsaicin, and ginger confered large benefit for pain scores (as assessed by WOMAC and KOOS pain scales) when compared to placebo.

\section{Pain efficacy compared to active comparator}

For pain, as assessed by VAS, NRS, and Likert pain scores, the overall SMD was 0.32 (95\% CI: -0.04 to $0.67 ; P=0.08)$, indicating no significant benefit for botanical therapies on pain scores (as assessed by VAS and NRS pain scales) when compared to an active comparator (Figure 3), and -0.08 ( $95 \%$ CI: -0.42 to $0.25 ; P=0.6$ ) for WOMAC/KOOS pain scores. There was significant heterogeneity between studies $\left(I^{2}=90.1 \%, P<0.001\right.$ [Figure 3]; $I^{2}=85.9 \%$ [Figure 4]) There was no association between the risk of bias and ES for either

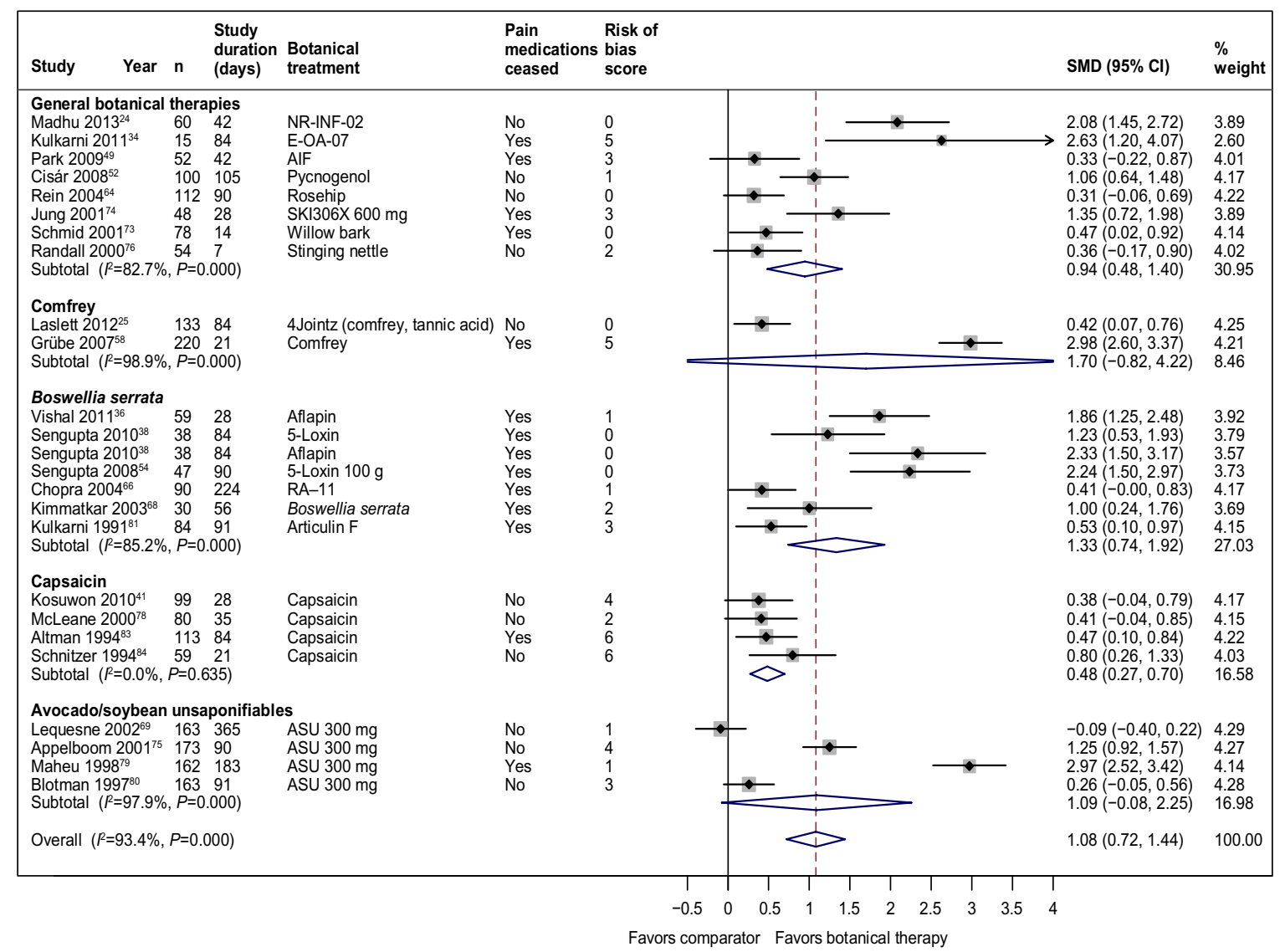

Figure I Efficacy of plant-derived therapies compared to placebo on VAS and NRS pain scores.

Note: Weights are from random effects analysis.

Abbreviations: $\mathrm{n}$, number of study participants; SMD, standardized mean difference; $\mathrm{Cl}$, confidence interval; VAS, visual analog scale; NRS, numeric rating scale; AIF, antiinflammatory factor; ASU, avocado/soybean unsaponifiables. 


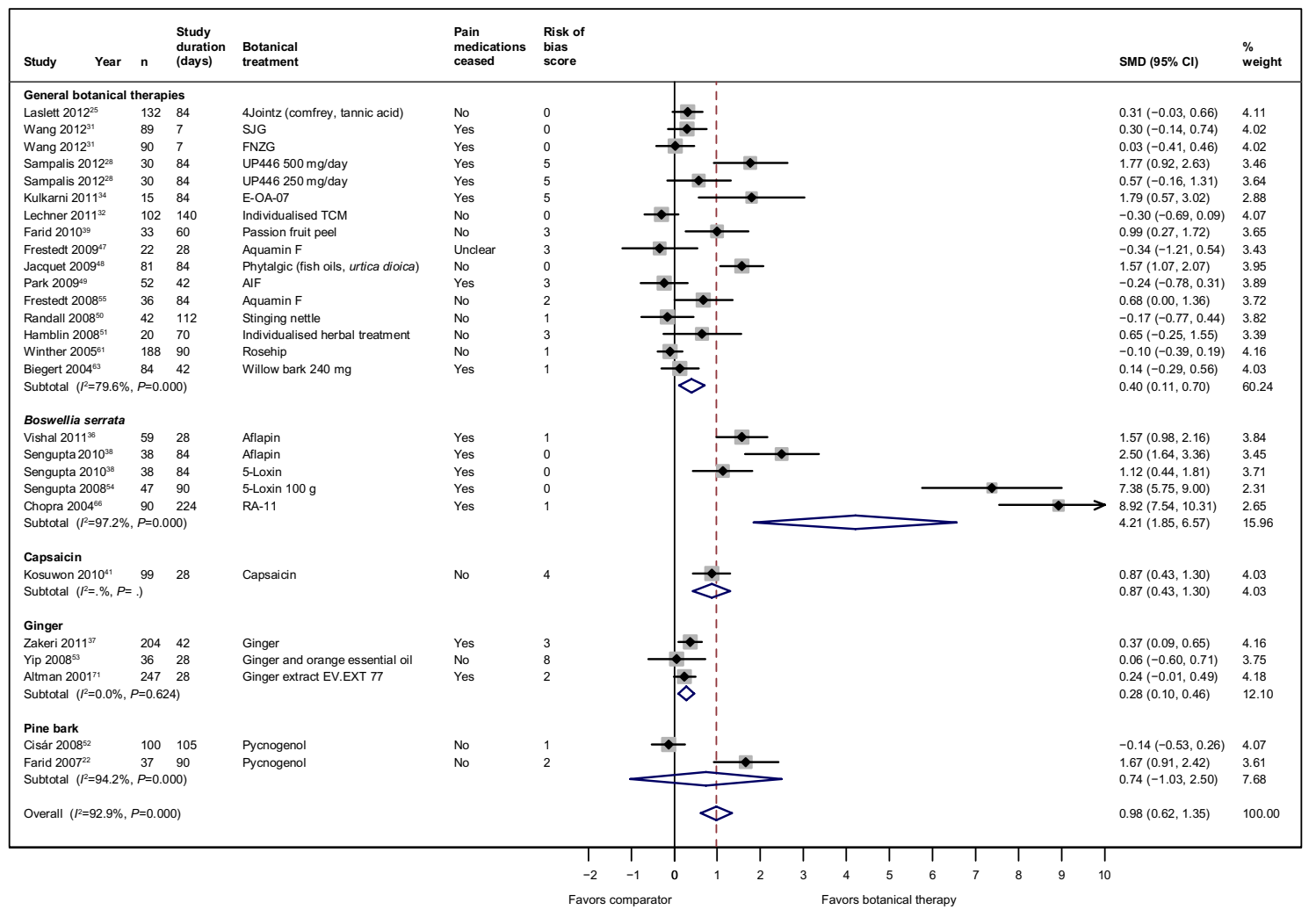

Figure 2 Efficacy of plant-derived therapies compared to placebo on pain WOMAC and KOOS pain scores.

Note: Weights are from random effects analysis.

Abbreviations: AIF, anti-inflammatory factor; n, number of study participants; SMD, standardized mean difference; Cl, confidence interval; FNZG, Fufang Nanxing Zhitong Gao; SJG, Shangshi Jietong Gao; AIF, anti-inflammatory factor; WOMAC, Western Ontario and McMaster Universities Osteoarthritis Index; KOOS, Knee injury and Osteoarthritis Outcome Score; TCM, traditional Chinese medicine.

the VAS and NRS pain scores or WOMAC/KOOS pain scores ( $\rho=-0.22 ; P=0.5 ; \rho=+0.30, P=0.4)$.

Only two trials demonstrated efficacy when compared to an active comparator: NR-INF-02 (containing curcumin) ${ }^{24}$ compared to glucosamine sulfate, $1,500 \mathrm{mg}$; and Harpadol ${ }^{\mathbb{R}}$ (Devil's claw), containing Harpagophytum procumbens, compared to diacerein, $100 \mathrm{mg}^{77}$ (Figure 3). Two therapies demonstrated significantly worse efficacy than active control: a Chinese herbal recipe (Duhuo Jisheng Wan) over 4 weeks; ${ }^{62}$ and willow bark extract (Salix daphnoides) over 42 days ${ }^{63}$ of treatment. Both studies used the same active control (diclofenac, $75 \mathrm{mg} /$ day or $100 \mathrm{mg} /$ day) (Figure 4).

\section{Function efficacy}

Function efficacy (when compared to placebo) was similar to that of the WOMAC/KOOS pain scores, with overall SMDs for function in plant-based therapies of 0.92 (95\% CI: $0.62-1.23 ; P \leq 0.001)$. The Boswellia trials demonstrated SMD of 1.66 (95\% CI: $0.77-2.55 ; P<0.001)$, ginger at 0.73 (95\% CI: -0.23 to $1.69 ; P=0.14)$, and ASUs at 1.10 (95\% CI: $0.17-1.21 ; P=0.021)$, with significant heterogeneity observed in all subgroups and for the therapies as a whole. There was no association between ES and risk of bias $(\rho=+0.01 ; P=0.96)$.

Agents demonstrating significant benefit over placebo include UP446 (250 mg/day and $500 \mathrm{mg} /$ day formulations $),{ }^{28}$ E-OA-07 (extract of shyonak, ashwagandha, shunthi, guggal, chopchini, rasana, and shallaki), ${ }^{34}$ passion fruit peel, ${ }^{39}$ SKI306X (extract of Clematis mandshurica, Trichosanthes kirilowii, and Prunella vulgaris), ${ }^{74}$ and NP 06-1 (Phellodendron amurense), but the effect was present only for overweight patients and absent in obese patients ${ }^{44}$ (data not shown).

Compounds containing Urtica dioica demonstrated benefit in one study, ${ }^{48}$ but not in another. ${ }^{76}$ Studies investigating the effect of $4 \mathrm{Jointz}^{\circledR}{ }^{25}$ seaweed, ${ }^{47,55}$ willow bark, ${ }^{63,73} \mathrm{AIF},{ }^{49}$ pine bark, ${ }^{22}$ and individualized herbal treatment ${ }^{51}$ did not demonstrate efficacy on function.

Overall, plant-based therapies demonstrated efficacy compared to placebo for OA function. Compared to the active comparator, the overall SMD was similar to that for WOMAC/ KOOS pain scores, at -0.04 (95\% CI: -0.40 to $0.32 ; P=0.99$ ), indicating no difference between the efficacy of botanical therapies and active comparator on function scores, but 
Table 3 Summary of efficacy findings of plant-based therapy compared to placebo, by therapy class

\begin{tabular}{|c|c|c|c|}
\hline Class of plant-based therapy & $\begin{array}{l}\text { VAS/NRS } \\
\text { pain score }\end{array}$ & $\begin{array}{l}\text { WOMACI } \\
\text { KOOS pain }\end{array}$ & $\begin{array}{l}\text { WOMACI } \\
\text { KOOS function }\end{array}$ \\
\hline ASU $69,75,79,80$ & No & - & Yes \\
\hline Boswellia serrata ${ }^{36,38,54,59,60,66,68,81}$ & Yes & Yes & Yes \\
\hline Capsaicin ${ }^{41,78,83,84}$ & Yes & Yes & Yes \\
\hline Comfrey ${ }^{25,58}$ & No & (No) & (No) \\
\hline Ginger ${ }^{37,53,71}$ & - & Yes & No \\
\hline Pycnogenol $^{22,52}$ & (Yes) & No & (Yes) \\
\hline \multicolumn{4}{|l|}{ Other treatments } \\
\hline Rosehip ${ }^{61,64}$ & (No) & (No) & - \\
\hline Stinging nettle $\mathrm{e}^{50,76}$ & (No) & (No) & (No) \\
\hline Willow bark 63,73 & (Yes) & (No) & No \\
\hline NR-INF-02 ${ }^{24}$ & Yes & - & - \\
\hline UP446 $250 \mathrm{mg}^{28}$ & - & No & Yes \\
\hline UP446 $500 \mathrm{mg}^{28}$ & - & Yes & Yes \\
\hline $\mathrm{FNZG/SJG} \mathrm{G}^{31}$ & - & No & - \\
\hline Individualized TCM/nonspecific herbal treatment ${ }^{32}$ & - & No & - \\
\hline $\mathrm{E}-\mathrm{OA}-07^{34}$ & Yes & Yes & Yes \\
\hline Passion fruit pee $\left.\right|^{39}$ & - & Yes & \\
\hline Phellodendron and citrus extracts (NP 06-I $)^{44}$ & - & - & No \\
\hline Phytalgic ${ }^{48}$ & - & Yes & Yes \\
\hline $\operatorname{AlF}^{49}$ & No & - & No \\
\hline Individualized herbal treatment ${ }^{51}$ & - & No & No \\
\hline Aquamin $\mathrm{F}^{47,55}$ & - & Yes & No \\
\hline SKI306X $\mathrm{X}^{74}$ & Yes & - & Yes \\
\hline
\end{tabular}

Notes: Status in brackets indicates that data are only available for one trial within the class. Rosehip, stinging nettle, and willow bark were all trialed in two studies, but they are not directly comparable on the same outcome measure.

Abbreviations: VAS, visual analog scale; NRS, numeric rating scale; WOMAC, Western Ontario and McMaster Universities Osteoarthritis Index; KOOS, Knee injury and Osteoarthritis Outcome Score; ASU, avocado/soybean unsaponifiables; FNZG, Fufang Nanxing Zhitong Gao; SJG, Shangshi Jietong Gao; TCM, traditional Chinese medicine; AlF, anti-inflammatory factor.

there is significant heterogeneity between studies $\left(I^{2}=92.7 \%\right.$; $P<0.001)$. Botanical therapies that demonstrate efficacy when compared to an active comparator are olive oil ${ }^{29}$ and UP446 ${ }^{28}$ (both low and high doses). Du huo ji sheng wan ${ }^{62}$ and willow bark $^{63}$ favored the active comparator. There was no association between $\mathrm{ES}$ and risk of bias $(\rho=0.2 ; P=0.44)$.

\section{Safety}

Figure 5 shows that the RR of one or more adverse events was not increased among patients receiving botanical therapies compared to placebo ( $\mathrm{RR}=1.13$; 95\% CI: $0.98-1.31$; $P=0.10)$ using a random effects model, with significant heterogeneity $(P=0.050)$, and $I^{2}$ of $28.0 \%$. Ginger and capsaicin were associated with increase risk of adverse events: the RR for ginger is 1.40 (95\% CI: $1.09-1.80, P=0.009)$, and the RR for capsaicin is 5.59 (95\% CI 2.92-10.69; $P<0.001)$. This is attributable to gastrointestinal events in the largest ginger trial ${ }^{71}$ and a localized burning sensation at the site of application for capsaicin. No trials other than those using capsaicin demonstrated an increased risk of adverse events compared to placebo. Reporting was often inadequate, with underreporting of adverse events common, particularly for adverse events that the investigators considered as not related to the study drug.

Figure 6 shows that the RR of one or more adverse events was reduced among patients receiving botanical therapies compared to an active comparator $(\mathrm{RR}=0.75$; 95\% CI: $0.65-0.85 ; P<0.001)$ using a fixed effects model, with no heterogeneity $(P=0.4)$, and $I^{2}$ of $3.3 \%$. Only one individual trial demonstrated reduced risk of adverse events compared to the active comparator; ${ }^{77}$ where Harpadol (Harpagophytum procumbens) reduced the risk of adverse events when compared to diacerein $100 \mathrm{mg} /$ day over 4 months of treatment. A reduction in adverse events was primarily found to be the reduction in gastrointestinal side effects when compared to nonsteroidal anti-inflammatory medications.

\section{Discussion}

This review compared the effects of plant-derived therapies from randomized controlled trials, when compared to placebo or an active comparator, on osteoarthritic pain and function. The efficacy of plant-derived therapies is superior to placebo and comparable to active comparators for treating 


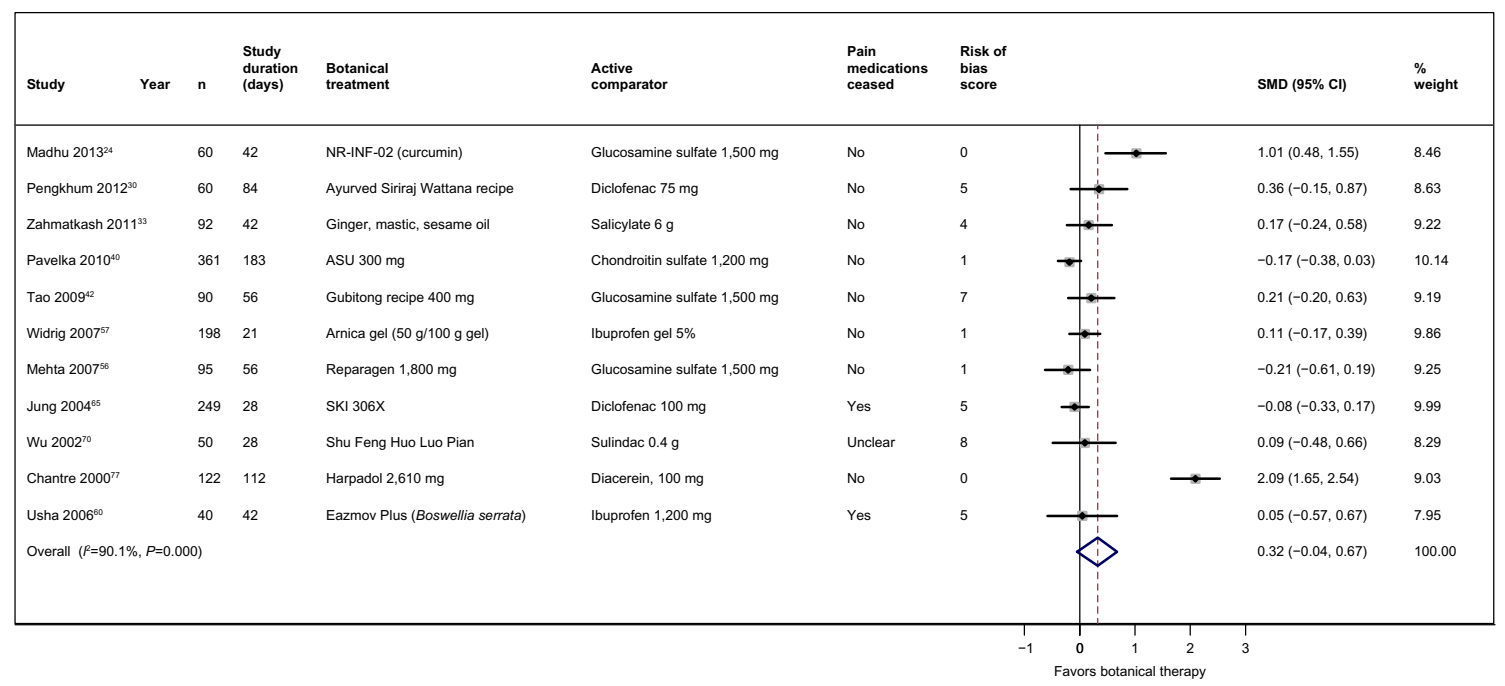

Figure 3 Efficacy of plant-derived therapies compared to active comparator on VAS and NRS pain scores.

Note: Weights are from random effects analysis.

Abbreviations: ASU, avocado/soybean unsaponifiables; n, number of study participants; SMD, standardized mean differences; Cl, confidence interval; VAS, visual analog scale; NRS, numeric rating scale.

osteoarthritic pain and functional limitations. Risk of one or more adverse events is not increased with the use of plantbased therapies when compared to placebo, but the risk is decreased by $25 \%$ when compared to an active comparator. Therefore, plant-derived therapies have a favorable risk profile compared to standard osteoarthritic therapies.

We observed significant heterogeneity for both pain and functional outcomes. This is expected, as these plant-derived therapies contain a wide variety of active ingredients and, therefore, potentially therapeutically active molecules. ${ }^{20}$ However, heterogeneity exists within classes, which is not explained by differences in the chemical components of treatments. The trials of ASUs ${ }^{69,75,79,80}$ have SMDs with a very wide range. The trial by Lequesne et $\mathrm{a}^{69}$ is the longest at 12 months' duration, with others trialed over 3 months ${ }^{75,80}$ or 6 months' durations. ${ }^{79}$ This may indicate that ASU is not efficacious over longer periods of time for osteoarthritic knee pain. Several trials of Boswellia serrata $a^{36,38,54,59,60,66,68,81}$ have

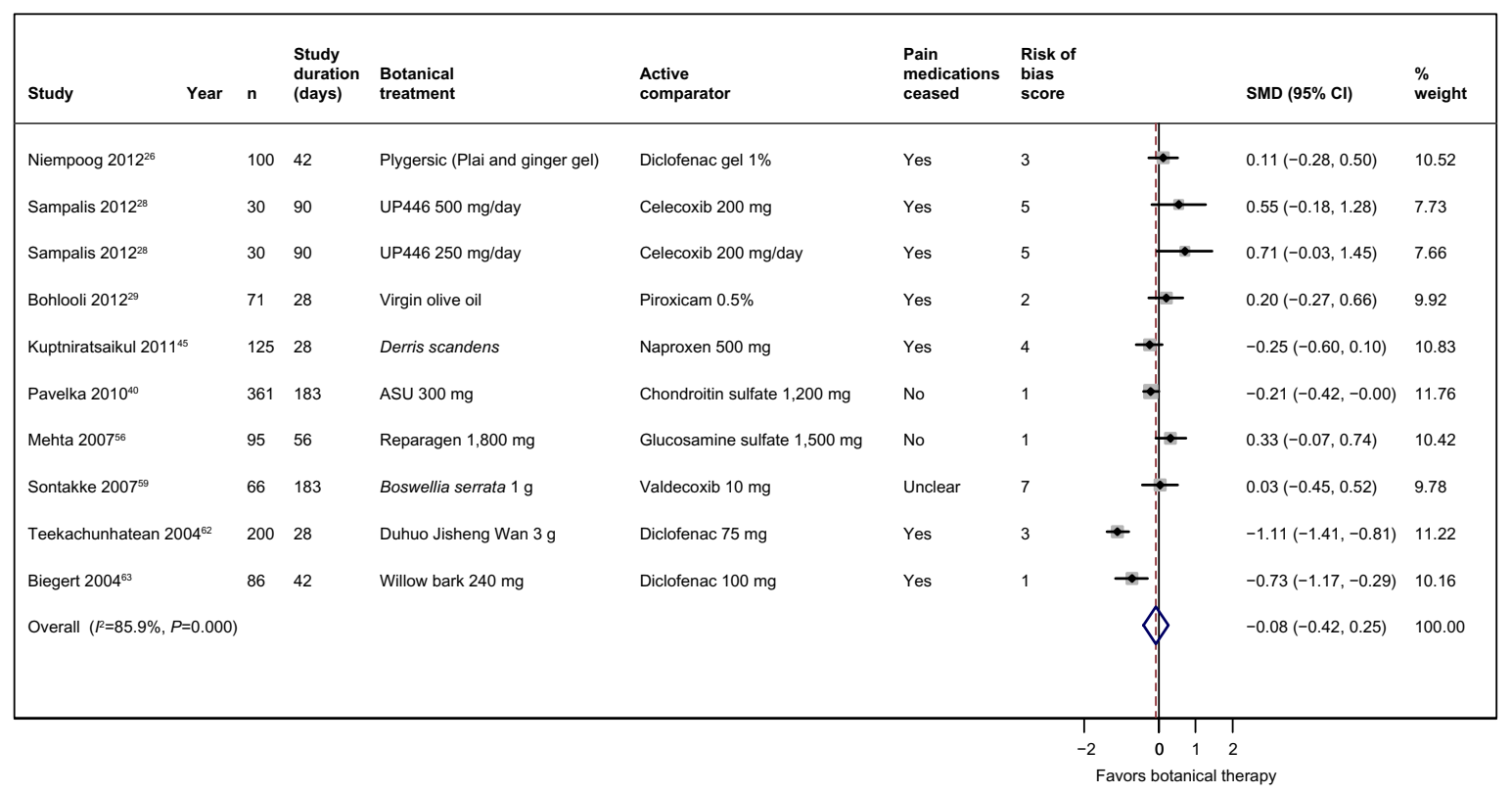

Figure 4 Efficacy of plant-derived therapies compared to active comparator on WOMAC and KOOS pain scores.

Note: Weights are from random effects analysis.

Abbreviations: ASU, avocado/soybean unsaponifiables; n, number of study participants; SMD, standardized mean differences; CI, confidence interval; WOMAC, Western Ontario and McMaster Universities Osteoarthritis Index; KOOS, Knee injury and Osteoarthritis Outcome Score. 


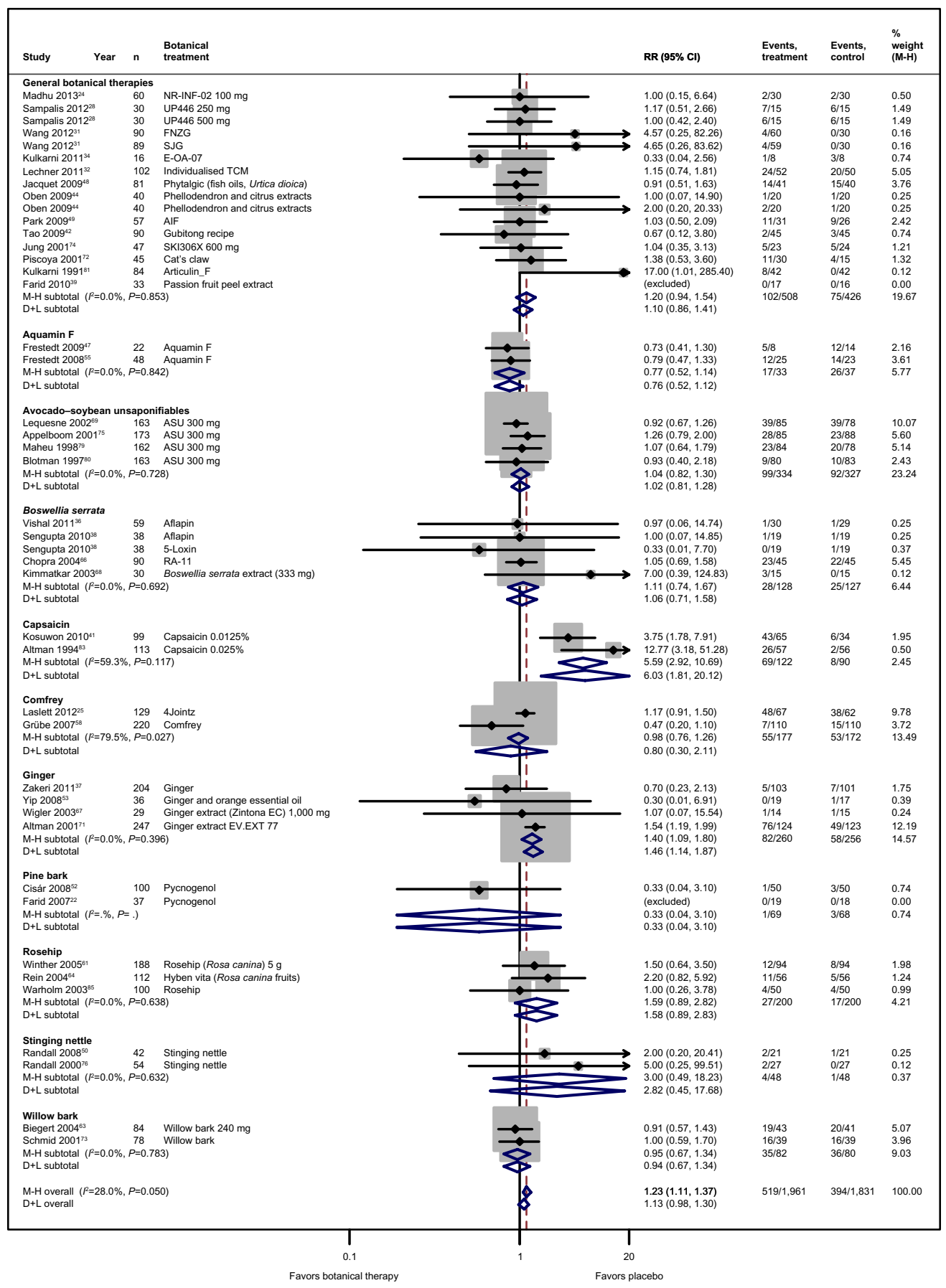

Figure 5 Safety of plant-derived therapies compared to placebo: incidence of one or more adverse events.

Abbreviations: n, number of study participants; FNZG, Fufang Nanxing Zhitong Gao; SJG, Shangshi Jietong Gao; RR, relative risk; Cl, confidence interval; AIF, antiinflammatory factor; TCM, traditional Chinese medicine; $\mathrm{M}-\mathrm{H}$, effect size for the risk ratio using a fixed effect model using the method of Mantel and Haenszel; D+L, effect size for the risk ratio using a random effects model using the method of DerSimonian and Laird.

extremely large ES. All trials in this class required study participants to cease pain medications before the trials commenced, possibly increasing the likelihood of demonstrating an effect of the plant therapy. None of the trials in this class was classified as being at high risk of bias in any category, although the risk of bias was unclear in numerous domains in several trials.
Among the four included trials of topical capsaicin, ${ }^{41,78,83,84}$ all had high risk of bias in one subgroup, three had incomplete outcome data (attrition bias), and one exhibited allocation concealment. The use of capsaicin is associated with an increased risk of adverse events $(R R=5.6)$, primarily a burning sensation at the site of application, which is of mild intensity and 


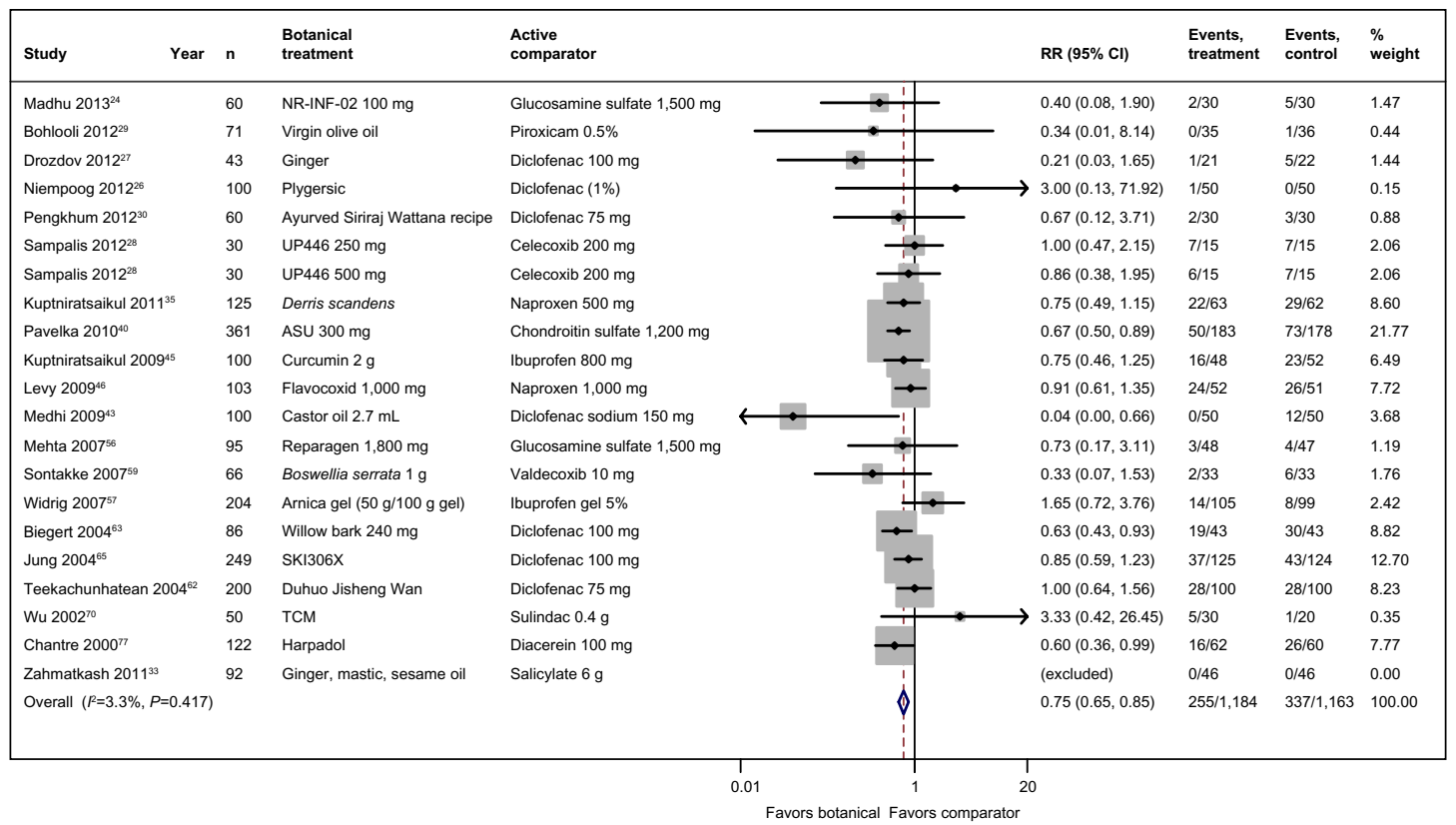

Figure 6 Safety of botanical therapy compared to active comparator: incidence of one or more adverse events.

Abbreviations: $n$, number of study participants; RR, relative risk; $\mathrm{Cl}$, confidence interval; $\mathrm{ASU}$, avocado/soybean unsaponifiables; TCM, traditional Chinese medicine.

diminishes with continued use ${ }^{83,92}$ However, this common adverse event makes allocation concealment of capsaicin trials challenging.

The ES (in SMDs) of many of the trials is very large, exceeding 1 . While this is technically and methodologically possible, some of the exceedingly large ES are unexpectedly and implausibly large. Statistically significant correlations between study quality and ES have been reported in other settings, but we did not observe that in this review. Most studies included estimates of variation (either SD or standard errors) of baseline and follow-up measurements, but not change scores, with over $60 \%$ of studies not having SD or standard errors for change scores. Therefore, correlations between baseline and the last follow-up have been estimated conservatively at $r=0.7$. This underestimates the ES if the true correlation is larger than this and overestimates it if the true correlation is smaller. For example, the SMD for VAS/ NRS pain scores for the Boswellia class is 1.33 with the existing assumption ( $r=0.7), 2.03$ (95\% CI: 1.01-3.06) with a higher correlation ( $r=0.95$ ), and 1.13 (95\% CI: 0.67-1.59) with a lower correlation $(r=0.5)$ - though botanical therapy is favored over placebo in all three scenarios. In these scenarios, SMD for VAS/NRS pain scores overall are 1.62 (95\% CI: 1.10-2.14; $r=0.95$ ), 0.94 (95\% CI: 0.62-1.26; $r=0.5$ ), and 0.86 (95\% CI: $0.57-1.15 ; r=0.3)$. Correlations are also likely to vary between treatment, placebo, and active control groups and they may be different depending on the intervention studied. Overall, estimates in this review may be underestimates of efficacy if the correlation is greater than this and they may be overestimates if the correlation is less. Reporting of the SD or standard error of the mean of the change in outcomes is required for more precise pooling of study data in future reviews.

Many therapies are only trialed in one clinical trial, or in only one trial for an individual outcome. First, this suggests that additional studies are required to validate the findings, and second, this makes pooling therapies difficult or impossible. This is especially so when treatments are compared to an active comparator but this is more broadly applicable. Numerous studies were poorly described, with only 12 studies having a low risk of bias in all dimensions, and only a further 12 studies scored as having a risk of bias that was unclear in only one dimension. Conduct of the trial (or reporting of the conduct of the trial) in the remaining studies was unclear regarding the risk of bias in more than one domain, and $31 \%$ were at high risk of bias in one or more domains. Use of the Consolidated Standards of Reporting Trials statement for the reporting of new clinical trials will be a welcome development.

\section{Conclusion}

Plant-derived therapies may be efficacious in treating osteoarthritic pain and functional limitation when compared to placebo, and similarly effective when compared to active comparators. The safety profile is similar to placebo and better than active comparators. However, quality trials and 
long-term data are lacking, and the number of trials for each therapy is limited. A comparison of efficacy would be assisted by trial standardization.

\section{Acknowledgments}

The authors would like to thank Dr P Rohdewald, Dr J Frestedt, and Dr Richard Rosenbloom for providing additional unpublished information on their clinical trials.

\section{Disclosure}

Dr Laslett is supported by an Arthritis Australia Heald Fellowship and a National Health and Medical Research Council Early Career Fellowship. Dr Jin is supported by an International Postgraduate Research Scholarship. Prof Graeme Jones is supported by a National Health and Medical Research Council Practitioner Fellowship. The authors' institution received funding for a study included in this review (Laslett 2012) from Arthritis Relief Plus Pty Ltd, but the authors report no other conflicts of interest in this work.

\section{References}

1. Vos T, Flaxman AD, Naghavi M, et al. Years lived with disability (YLDs) for 1160 sequelae of 289 diseases and injuries 1990-2010: a systematic analysis for the Global Burden of Disease Study 2010. Lancet. 2012;380(9859):2163-2196.

2. Murray CJ, Vos T, Lozano R, et al. Disability-adjusted life years (DALYs) for 291 diseases and injuries in 21 regions, 1990-2010: a systematic analysis for the Global Burden of Disease Study 2010. Lancet. 2012;380(9859):2197-2223.

3. Lane NE, Brandt K, Hawker G, et al. OARSI-FDA initiative: defining the disease state of osteoarthritis. Osteoarthritis Cartilage. 2011;19(5): $478-482$.

4. Loeser RF, Goldring SR, Scanzello CR, Goldring MB. Osteoarthritis: a disease of the joint as an organ. Arthritis Rheum. 2012;64(6): 1697-1707.

5. Dieppe PA, Lohmander LS. Pathogenesis and management of pain in osteoarthritis. Lancet. 2005;365(9463):965-973.

6. Jones G. Osteoarthritis: Where are we for pain and therapy in 2013 ? Aust Fam Physician. 2013;42(11):766-769.

7. Kidd BL, Morris VH, Urban L. Pathophysiology of joint pain. Ann Rheum Dis. 1996;55(5):276-283.

8. Schaible HG, Grubb BD. Afferent and spinal mechanisms of joint pain. Pain. 1993;55(1):5-54.

9. Zhang W, Nuki G, Moskowitz RW, et al. OARSI recommendations for the management of hip and knee osteoarthritis: part III: Changes in evidence following systematic cumulative update of research published through January 2009. Osteoarthritis Cartilage. 2010;18(4): 476-499.

10. Tubach F, Ravaud P, Martin-Mola E, et al. Minimum clinically important improvement and patient acceptable symptom state in pain and function in rheumatoid arthritis, ankylosing spondylitis, chronic back pain, hand osteoarthritis, and hip and knee osteoarthritis: Results from a prospective multinational study. Arthritis Care Res (Hoboken). 2012;64(11):1699-1707.

11. Bombardier C, Laine L, Reicin A, et al; VIGOR Study Group. Comparison of upper gastrointestinal toxicity of rofecoxib and naproxen in patients with rheumatoid arthritis. VIGOR Study Group. $N$ Engl $J$ Med. 2000;343(21):1520-1528, 2 p following 1528.
12. Bresalier RS, Sandler RS, Quan H, et al; Adenomatous Polyp Prevention on Vioxx (APPROVe) Trial Investigators. Cardiovascular events associated with rofecoxib in a colorectal adenoma chemoprevention trial. N Engl J Med. 2005;352(11):1092-1102.

13. Caughey GE, Roughead EE, Pratt N, Killer G, Gilbert AL. Stroke risk and NSAIDs: an Australian population-based study. Med J Aust. 2011;195(9):525-529.

14. Solomon SD, McMurray JJ, Pfeffer MA, et al; Adenoma Prevention with Celecoxib (APC) Study Investigators. Cardiovascular risk associated with celecoxib in a clinical trial for colorectal adenoma prevention. N Engl J Med. 2005;352(11):1071-1080.

15. McAlindon TE, Jacques P, Zhang Y, et al. Do antioxidant micronutrients protect against the development and progression of knee osteoarthritis? Arthritis Rheum. 1996;39(4):648-656.

16. Zochling J, March L, Lapsley H, Cross M, Tribe K, Brooks P. Use of complementary medicines for osteoarthritis - a prospective study. Ann Rheum Dis. 2004;63(5):549-554.

17. Quandt SA, Chen H, Grzywacz JG, Bell RA, Lang W, Arcury TA. Use of complementary and alternative medicine by persons with arthritis: results of the National Health Interview Survey. Arthritis Rheum. 2005;53(5):748-755.

18. Little CV, Parsons T. Herbal therapy for treating osteoarthritis [review]. Cochrane Database Syst Rev. 2001;1:CD002947.

19. Long L, Soeken K, Ernst E. Herbal medicines for the treatment of osteoarthritis: a systematic review. Rheumatology (Oxford). 2001;40(7): 779-793.

20. Chrubasik JE, Roufogalis BD, Chrubasik S. Evidence of effectiveness of herbal antiinflammatory drugs in the treatment of painful osteoarthritis and chronic low back pain. Phytother Res. 2007;21(7): 675-683.

21. De Silva V, El-Metwally A, Ernst E, Lewith G, Macfarlane GJ; Arthritis Research UK Working Group on Complementary and Alternative Medicines. Evidence for the efficacy of complementary and alternative medicines in the management of osteoarthritis: a systematic review. Rheumatology (Oxford). 2011;50(5):911-920.

22. Farid R, Mirfeizi Z, Mirheidari M, et al. Pycnogenol supplementation reduces pain and stiffness and improves physical function in adults with knee osteoarthritis. Nutrition Research. 2007;27(11):692-697.

23. The Cochrane Collaboration [homepage on the Internet]. The Cochrane Collaboration's tool for assessing risk of bias. The Cochrane Collaboration; 2011 [updated March 20, 2011; cited Jan 21, 2014]. Available from: http://handbook.cochrane.org/chapter_8/table_8_5_a_ the_cochrane_collaborations_tool_for_assessing.htm. Accessed Jan 21, 2014.

24. Madhu K, Chanda K, Saji MJ. Safety and efficacy of Curcuma longa extract in the treatment of painful knee osteoarthritis: a randomized placebo-controlled trial. Inflammopharmacology. 2013;21(2):129-136.

25. Laslett LL, Quinn SJ, Darian-Smith E, et al. Treatment with 4Jointz reduces knee pain over 12 weeks of treatment in patients with clinical knee osteoarthritis: a randomised controlled trial. Osteoarthritis Cartilage. 2012;20(11):1209-1216.

26. Niempoog S, Siriarchavatana P, Kajsongkram T. The efficacy of Plygersic gel for use in the treatment of osteoarthritis of the knee. J Med Assoc Thai. 2012;95 Suppl 10:S113-S119.

27. Drozdov VN, Kim VA, Tkachenko EV, Varvanina GG. Influence of a specific ginger combination on gastropathy conditions in patients with osteoarthritis of the knee or hip. JAltern Complement Med. 2012;18(6): 583-588.

28. Sampalis JS, Brownell LA. A randomized, double blind, placebo and active comparator controlled pilot study of UP446, a novel dual pathway inhibitor anti-inflammatory agent of botanical origin. Nutr $J$. 2012;11:21.

29. Bohlooli S, Jastan M, Nakhostin-Roohi B, Mohammadi S, Baghaei Z. A pilot double-blinded, randomized, clinical trial of topical virgin olive oil versus piroxicam gel in osteoarthritis of the knee. J Clin Rheumatol. 2012;18(2):99-101. 
30. Pengkhum T, Chatsiricharoenkul S, Akarasereenont $\mathrm{P}$, Charoencholvanich K. Phase II clinical trial of Ayurved Siriraj Wattana Recipe for symptomatic relief in patients with osteoarthritis of the knee. J Med Assoc Thai. 2012;95(3):452-460.

31. Wang X, Cao Y, Pang J, et al. Traditional chinese herbal patch for short-term management of knee osteoarthritis: a randomized, doubleblind, placebo-controlled trial. Evid Based Complement Alternat Med. 2012;2012:171706.

32. Lechner M, Steirer I, Brinkhaus B, et al. Efficacy of individualized Chinese herbal medication in osteoarthrosis of hip and knee: a doubleblind, randomized-controlled clinical study. JAltern Complement Med. 2011;17(6):539-547.

33. Zahmatkash M, Vafaeenasab MR. Comparing analgesic effects of a topical herbal mixed medicine with salicylate in patients with knee osteoarthritis. Pak J Biol Sci. 2011;14(13):715-719.

34. Kulkarni MP, Shakeel A, Shinde BS, Rosenbloom RA. Efficacy and safety of E-OA-07 in moderate to severe symptoms of osteoarthritis: a double-blind randomized placebo-controlled study. Am J Ther 2011;18(2):170-177.

35. Kuptniratsaikul V, Pinthong T, Bunjob M, Thanakhumtorn S, Chinswangwatanakul P, Thamlikitkul V. Efficacy and safety of Derris scandens Benth extracts in patients with knee osteoarthritis. J Altern Complement Med. 2011;17(2):147-153.

36. Vishal AA, Mishra A, Raychaudhuri SP. A double blind, randomized, placebo controlled clinical study evaluates the early efficacy of aflapin in subjects with osteoarthritis of knee. Int J Med Sci. 2011;8(7): 615-622.

37. Zakeri Z, Izadi S, Bari Z, Soltani F, Narouie B, Ghasemi-rad M. Evaluating the effects of ginger extract on knee pain, stiffness and difficulty in patients with knee osteoarthritis. Journal of Medicinal Plants Research. 2011;5(15):3375-3379.

38. Sengupta K, Krishnaraju AV, Vishal AA, et al. Comparative efficacy and tolerability of 5-Loxin and Aflapin Against osteoarthritis of the knee: a double blind, randomized, placebo controlled clinical study. Int J Med Sci. 2010;7(6):366-377.

39. Farid R, Rezaieyazdi Z, Mirfeizi Z, et al. Oral intake of purple passion fruit peel extract reduces pain and stiffness and improves physical function in adult patients with knee osteoarthritis. Nutr Res. 2010;30(9):601-606.

40. Pavelka K, Coste P, Géher P, Krejci G. Efficacy and safety of piascledine 300 versus chondroitin sulfate in a 6 months treatment plus 2 months observation in patients with osteoarthritis of the knee. Clin Rheumatol. 2010;29(6):659-670.

41. Kosuwon W, Sirichatiwapee W, Wisanuyotin T, Jeeravipoolvarn P, Laupattarakasem W. Efficacy of symptomatic control of knee osteoarthritis with $0.0125 \%$ of capsaicin versus placebo. J Med Assoc Thai. 2010;93(10):1188-1195.

42. Tao QW, Xu Y, Jin DE, Yan XP. Clinical efficacy and safety of Gubitong Recipe () in treating osteoarthritis of knee joint. Chin J Integr Med. 2009;15(6):458-461.

43. Medhi B, Kishore K, Singh U, Seth SD. Comparative clinical trial of castor oil and diclofenac sodium in patients with osteoarthritis. Phytother Res. 2009;23(10):1469-1473.

44. Oben J, Enonchong E, Kothari S, Chambliss W, Garrison R, Dolnick D. Phellodendron and Citrus extracts benefit joint health in osteoarthritis patients: a pilot, double-blind, placebo-controlled study. Nutr J. 2009;8:38.

45. Kuptniratsaikul V, Thanakhumtorn S, Chinswangwatanakul P, Wattanamongkonsil L, Thamlikitkul V. Efficacy and safety of Curcuma domestica extracts in patients with knee osteoarthritis. J Altern Complement Med. 2009;15(8):891-897.

46. Levy RM, Saikovsky R, Shmidt E, Khokhlov A, Burnett BP. Flavocoxid is as effective as naproxen for managing the signs and symptoms of osteoarthritis of the knee in humans: a short-term randomized, doubleblind pilot study. Nutr Res. 2009;29(5):298-304.

47. Frestedt JL, Kuskowski MA, Zenk JL. A natural seaweed derived mineral supplement (Aquamin F) for knee osteoarthritis: a randomised, placebo controlled pilot study. Nutr J. 2009;8:7.
48. Jacquet A, Girodet PO, Pariente A, Forest K, Mallet L, Moore N. Phytalgic, a food supplement, vs placebo in patients with osteoarthritis of the knee or hip: a randomised double-blind placebo-controlled clinical trial. Arthritis Res Ther. 2009;11(6):R192.

49. Park SH, Kim SK, Shin IH, Kim HG, Choe JY. Effects of AIF on Knee Osteoarthritis Patients: Double-blind, Randomized Placebo-controlled Study. Korean J Physiol Pharmacol. 2009;13(1):33-37.

50. Randall C, Dickens A, White A, Sanders H, Fox M, Campbell J. Nettle sting for chronic knee pain: a randomised controlled pilot study. Complement Ther Med. 2008;16(2):66-72.

51. Hamblin L, Laird A, Parkes E, Walker AF. Improved arthritic knee health in a pilot RCT of phytotherapy. J R Soc Promot Health. 2008; 128(5):255-262.

52. Cisár P, Jány R, Waczulíková I, et al. Effect of pine bark extract (Pycnogenol) on symptoms of knee osteoarthritis. Phytother Res. 2008;22(8):1087-1092.

53. Yip YB, Tam AC. An experimental study on the effectiveness of massage with aromatic ginger and orange essential oil for moderate-to-severe knee pain among the elderly in Hong Kong. Complement Ther Med. 2008;16(3):131-138.

54. Sengupta K, Alluri KV, Satish AR, et al. A double blind, randomized, placebo controlled study of the efficacy and safety of 5-Loxin for treatment of osteoarthritis of the knee. Arthritis Res Ther. 2008;10(4):R85.

55. Frestedt JL, Walsh M, Kuskowski MA, Zenk JL. A natural mineral supplement provides relief from knee osteoarthritis symptoms: a randomized controlled pilot trial. Nutr J. 2008;7:9.

56. Mehta K, Gala J, Bhasale S, et al. Comparison of glucosamine sulfate and a polyherbal supplement for the relief of osteoarthritis of the knee: a randomized controlled trial [ISRCTN25438351]. BMC Complement Altern Med. 2007;7:34.

57. Widrig R, Suter A, Saller R, Melzer J. Choosing between NSAID and arnica for topical treatment of hand osteoarthritis in a randomised, double-blind study. Rheumatol Int. 2007;27(6):585-591.

58. Grube B, Grünwald J, Krug L, Staiger C. Efficacy of a comfrey root (Symphyti offic. radix) extract ointment in the treatment of patients with painful osteoarthritis of the knee: results of a double-blind, randomised, bicenter, placebo-controlled trial. Phytomedicine. 2007;14(1):2-10.

59. Sontakke S, Thawani V, Pimpalkhute S, Kabra P, Babhulkar S, Hingorani L. Open, randomized, controlled clinical trial of Boswellia serrata extract as compared to valdecoxib in osteoarthritis of knee. Indian $J$ Pharmacol. 2007;39(1):27-29.

60. Usha PR, Naidu MUR. Clinical evaluation of eazmov plus in patients of osteoarthritis. Phytomedica. 2006;7:21-30.

61. Winther K, Apel K, Thamsborg G. A powder made from seeds and shells of a rose-hip subspecies (Rosa canina) reduces symptoms of knee and hip osteoarthritis: a randomized, double-blind, placebo-controlled clinical trial. Scand J Rheumatol. 2005;34(4):302-308.

62. Teekachunhatean S, Kunanusorn P, Rojanasthien N, et al. Chinese herbal recipe versus diclofenac in symptomatic treatment of osteoarthritis of the knee: a randomized controlled trial [ISRCTN70292892]. BMC Complement Altern Med. 2004;4:19.

63. Biegert C, Wagner I, Lüdtke R, et al. Efficacy and safety of willow bark extract in the treatment of osteoarthritis and rheumatoid arthritis: results of 2 randomized double-blind controlled trials. $J$ Rheumatol. 2004;31(11):2121-2130.

64. Rein E, Kharazmi A, Winther K. A herbal remedy, Hyben Vital (stand. powder of a subspecies of Rosa canina fruits), reduces pain and improves general wellbeing in patients with osteoarthritis - a doubleblind, placebo-controlled, randomised trial. Phytomedicine. 2004;11(5): 383-391.

65. Jung YB, Seong SC, Lee MC, et al. A four-week, randomized, doubleblind trial of the efficacy and safety of SKI306X: a herbal anti-arthritic agent versus diclofenac in osteoarthritis of the knee. Am J Chin Med. 2004;32(2):291-301.

66. Chopra A, Lavin P, Patwardhan B, Chitre D. A 32-week randomized, placebo-controlled clinical evaluation of RA-11, an Ayurvedic drug, on osteoarthritis of the knees. J Clin Rheumatol. 2004;10(5):236-245. 
67. Wigler I, Grotto I, Caspi D, Yaron M. The effects of Zintona EC (a ginger extract) on symptomatic gonarthritis. Osteoarthritis Cartilage. 2003;11(11):783-789.

68. Kimmatkar N, Thawani V, Hingorani L, Khiyani R. Efficacy and tolerability of Boswellia serrata extract in treatment of osteoarthritis of knee - a randomized double blind placebo controlled trial. Phytomedicine. 2003;10(1):3-7.

69. Lequesne M, Maheu E, Cadet C, Dreiser RL. Structural effect of avocado/soybean unsaponifiables on joint space loss in osteoarthritis of the hip. Arthritis Rheum. 2002;47(1):50-58.

70. Wu X, Zhou Y. Observation on the therapeutic effect of Shu Feng Huo Luo Pian for treatment of osseous arthritis. J Tradit Chin Med. 2002;22(1):12-14.

71. Altman RD, Marcussen KC. Effects of a ginger extract on knee pain in patients with osteoarthritis. Arthritis Rheum. 2001;44(11):2531-2538.

72. Piscoya J, Rodriguez Z, Bustamante SA, Okuhama NN, Miller MJ, Sandoval M. Efficacy and safety of freeze-dried cat's claw in osteoarthritis of the knee: mechanisms of action of the species Uncaria guianensis. Inflamm Res. 2001;50(9):442-448.

73. Schmid B, Lüdtke R, Selbmann HK, et al. Efficacy and tolerability of a standardized willow bark extract in patients with osteoarthritis: randomized placebo-controlled, double blind clinical trial. Phytother Res. 2001;15(4):344-350.

74. Jung YB, Roh KJ, Jung JA, et al. Effect of SKI 306X, a new herbal anti-arthritic agent, in patients with osteoarthritis of the knee: a double-blind placebo controlled study. Am J Chin Med. 2001;29(3-4): 485-491.

75. Appelboom T, Schuermans J, Verbruggen G, Henrotin Y, Reginster JY. Symptoms modifying effect of avocado/soybean unsaponifiables (ASU) in knee osteoarthritis. A double blind, prospective, placebo-controlled study. Scand J Rheumatol. 2001;30(4):242-247.

76. Randall C, Randall H, Dobbs F, Hutton C, Sanders H. Randomized controlled trial of nettle sting for treatment of base-of-thumb pain. J R Soc Med. 2000;93(6):305-309.

77. Chantre P, Cappelaere A, Leblan D, Guedon D, Vandermander J, Fournie B. Efficacy and tolerance of Harpagophytum procumbens versus diacerhein in treatment of osteoarthritis. Phytomedicine. 2000;7(3):177-183.

78. McCleane G. The analgesic efficacy of topical capsaicin is enhanced by glyceryl trinitrate in painful osteoarthritis: a randomized, double blind, placebo controlled study. Eur J Pain. 2000;4(4):355-360.

79. Maheu E, Mazières B, Valat JP, et al. Symptomatic efficacy of avocado/ soybean unsaponifiables in the treatment of osteoarthritis of the knee and hip: a prospective, randomized, double-blind, placebo-controlled, multicenter clinical trial with a six-month treatment period and a twomonth followup demonstrating a persistent effect. Arthritis Rheum. 1998;41(1):81-91.
80. Blotman F, Maheu E, Wulwik A, Caspard H, Lopez A. Efficacy and safety of avocado/soybean unsaponifiables in the treatment of symptomatic osteoarthritis of the knee and hip. A prospective, multicenter, three-month, randomized, double-blind, placebo-controlled trial. Rev Rhum Engl Ed. 1997;64(12):825-834.

81. Kulkarni RR, Patki PS, Jog VP, Gandage SG, Patwardhan B. Treatment of osteoarthritis with a herbomineral formulation: a double-blind, placebo-controlled, cross-over study. J Ethnopharmacol. 1991;33(1-2):91-95.

82. Ferraz MB, Pereira RB, Iwata NM, Atra E. Tipi. A popular analgesic tea: a double-blind cross-over trial in osteoarthritis. Clin Exp Rheumatol. 1991;9(2):205-206.

83. Altman RD, Aven A, Holmburg CE, Pfeifer LM, Sack M, Young GT. Capsaicin cream $0.025 \%$ as Monotherapy for Osteoarthritis: A doubleblind study. Semin Arthritis Rheum. 1994;23(6):S25-S33.

84. Schnitzer T, Morton C, Coker S. Topical capsaicin therapy for osteoarthritis pain: Achieving a maintenance regimen. Semin Arthritis Rheum. 1994;23(6):34-40.

85. Warholm O, Skaar S, Hedman E, Mølmen HM, Eik L. The Effects of a Standardized Herbal Remedy Made from a Subtype of Rosa canina in Patients with Osteoarthritis: A Double-Blind, Randomized, PlaceboControlled Clinical Trial. Curr Ther Res Clin Exp. 2003;64(1): 21-31.

86. Bellamy N, Buchanan WW, Goldsmith CH, Campbell J, Stitt LW. Validation study of WOMAC: a health status instrument for measuring clinically important patient relevant outcomes to antirheumatic drug therapy in patients with osteoarthritis of the hip or knee. J Rheumatol. 1988;15(12):1833-1840.

87. Roos EM, Roos HP, Lohmander LS, Ekdahl C, Beynnon BD. Knee Injury and Osteoarthritis Outcome Score (KOOS) - development of a self-administered outcome measure. J Orthop Sports Phys Ther. 1998;28(2):88-96.

88. Davis AM, Badley EM, Beaton DE, et al. Rasch analysis of the Western Ontario McMaster (WOMAC) Osteoarthritis Index: results from community and arthroplasty samples. J Clin Epidemiol. 2003;56(11): 1076-1083.

89. Wolfe F, Kong SX. Rasch analysis of the Western Ontario MacMaster questionnaire (WOMAC) in 2205 patients with osteoarthritis, rheumatoid arthritis, and fibromyalgia. Ann Rheum Dis. 1999;58(9):563-568.

90. Lequesne MG, Mery C, Samson M, Gerard P. Indexes of severity for osteoarthritis of the hip and knee. Validation - value in comparison with other assessment tests. Scand J Rheumatol Suppl. 1987;65:85-89.

91. DerSimonian R, Laird N. Meta-analysis in clinical trials. Control Clin Trials. 1986;7(3):177-188.

92. Schnitzer TJ, Pelletier JP, Haselwood DM, et al. Civamide cream $0.075 \%$ in patients with osteoarthritis of the knee: a 12 -week randomized controlled clinical trial with a longterm extension. J Rheumatol. 2012;39(3):610-620.
Botanics: Targets and Therapy

\section{Publish your work in this journal}

Botanics: Targets and Therapy is an international, peer-reviewed, open access journal focusing on the discovery and development of active compounds based upon or found naturally occurring in the plant kingdom that may have therapeutic potential in any disease state. The manuscript management system is completely online and includes a very

\section{Dovepress}

quick and fair peer-review system. Visit http://www.dovepress.com/ testimonials.php to read real quotes from published authors. 